\section{Spaceborne Reflector SAR Systems with Digital Beamforming}

\author{
SIGURD HUBER \\ MARWAN YOUNIS, Senior Member, IEEE \\ ANTON PATYUCHENKO \\ GERHARD KRIEGER, Senior Member, IEEE \\ ALBERTO MOREIRA, Fellow, IEEE
}

Spaceborne synthetic aperture radar (SAR) imaging enters an era where increasingly short revisit times, or large swath widths, respectively, and high spatial resolutions are requested. These requirements impose contradicting constraints on conventional SAR systems using analog beamforming technology. The development for future radar satellites is therefore towards digital beamforming (DBF) systems where the analogous receiver hardware is replaced by digital components. Concerning the SAR antenna the innovative concept of a parabolic mesh reflector in conjunction with a digital feed array is becoming a promising architecture for this new SAR system generation. These antennas, already a mature technique for communication satellites, have the potential to outperform planar array antennas in terms of gain at a moderate hardware effort. This article provides a hardware concept study based on a design in X-band. Focus is put on DBF algorithms adopted to the SAR case and important performance figures are derived.
Manuscript received April 6, 2011; revised August 29, 2011; released for publication January 27, 2012.

IEEE Log No. T-AES/48/4/944216.

Refereeing of this contribution was handled by M. Akella.

Authors' address: Microwaves and Radar Institute, German Aerospace Center (DLR), Oberpfaffenhofen, Germany. E-mail: (sigurd.huber@dlr.de).

0018-9251/12/\$26.00 (c) 2012 IEEE

\section{INTRODUCTION}

Radar remote sensing is a technique which has been vastly exploited in spaceborne Earth observation applications in the last decades. With synthetic aperture radar (SAR) systems information about the reflectivity of distant objects can be retrieved. Those imaging radar systems typically consist of a transmitter which illuminates a certain part of the Earth surface, the footprint, and a receiver which collects the scattered electromagnetic energy. This signal is digitized and downlinked to a ground station.

Future applications like Earth system dynamics monitoring [1] require a short revisit time which in turn rises the need of large swath widths. At the same time operators and investigators are interested in a high information content of the SAR signal. This is traditionally achieved by increasing the signal bandwidth resulting in a higher resolution of the SAR images. Large swath widths and high resolutions are contradicting requirements for conventional SAR systems. Since spaceborne SAR systems demand a very high isolation between the transmit- and receive-channel, they cannot be implemented as $\mathrm{CW}$ radars on a single platform. This technical constraint leaves as the only option pulsed radar systems which are subject to a stringent timing of transmit and receive events. Aiming at high resolutions a broad footprint or, respectively, a broad antenna beam is required. In order to sample the received signal adequately, a sufficiently high pulse repetition frequency (PRF) has to be used. The high PRF in turn limits the swath width.

One possibility to overcome this restriction is to transmit a signal using a broad beam and to record with multiple receivers. These individual receiver signals are then processed in order to reconstruct the high resolution image. In terms of system theory such a SAR configuration would be a single input multiple output (SIMO) system. Classical approaches employ planar array antennas where the aperture is split on receive into multiple subapertures [2]. The performance for a spaceborne SAR system consisting of several formation-flying small satellites and the implications on signal processing have been investigated in [3]. The novel concept of combining a reflector antenna with a feed array, where the signal is digitized almost immediately after the receiver, was first proposed in [4] and further investigated in [5]-[10]. It was demonstrated that such systems have the potential to outperform conventional planar SAR systems. Reflector antenna based systems are already a mature technique for communication satellites. Generally reflector antennas inherently generate a shaped beam due to the mechanical molding of the reflector dish. That means for any given feed position only a certain solid angle can be illuminated. Therefore a set of multiple feed elements is required 
in order to cover the complete region of interest. By moving the analog-to-digital converters (ADCs) closer to the RF front end, it is possible to form beams by means of digital signal processing avoiding a costly analog receiver chain. It is the objective of this article to present a detailed analysis of this innovative hardware concept in the context of SAR and to discuss the performance of such systems. Emphasis is laid on digital beamforming (DBF) procedures which improve or balance the system performance.

The article is organized as follows. Starting with an overview of the system hardware concept in Section II-A, the reflector antenna design with feed array is presented in Section II-B and the system operation is outlined in Section III. Based on the SAR signal model presented in Section IV-A the DBF concepts are divided in elevation and azimuth. The corresponding DBF algorithms are derived in Sections IV-B and IV-C, respectively. These beamforming procedures are demonstrated by means of numerical simulations in Sections V-A and V-B. Conclusions are presented in Section VI.

\section{THE SAR SYSTEM}

In the following sections the basic hardware concepts of multi-channel reflector SAR systems are presented. In more detail the idea of combining a reflector antenna with a digital feed array, based on a design in X-band, is discussed.

\section{A. Hardware Architecture}

SAR systems employing a parabolic reflector antenna in conjunction with a feed array are an interesting alternative to conventional planar array antenna concepts. Parabolic reflector antennas differ from planar arrays insofar as they focus an incident plane wave in a compact zone, while planar arrays are illuminated homogeneously. Consequently, depending on the incidence angle of the wave, the energy has to be collected by means of a feed array. The main advantage over planar array antennas is the fact that high gain antennas can be easily realized by means of large light-weight foldable mesh reflectors. Figure 1 shows the basic concept of such a reflector-based SAR system [4]. The sensor flight direction is associated with the azimuth dimension in SAR coordinates. The elevation direction is in the paper plane associated with slant range. The lower part of the image shows the feed array. Every feed element, connected with a transmit/receive (T/R) module on transmit $(\mathrm{Tx})$, illuminates, after reflection from the reflector, a certain slightly overlapping angular domain in elevation. The received signals $u_{i}$ are digitized with ADCs and further processed in the DBF unit. Here hardware components such as low noise amplifiers (LNAs), T/R modules, mixers, filters, etc. are not
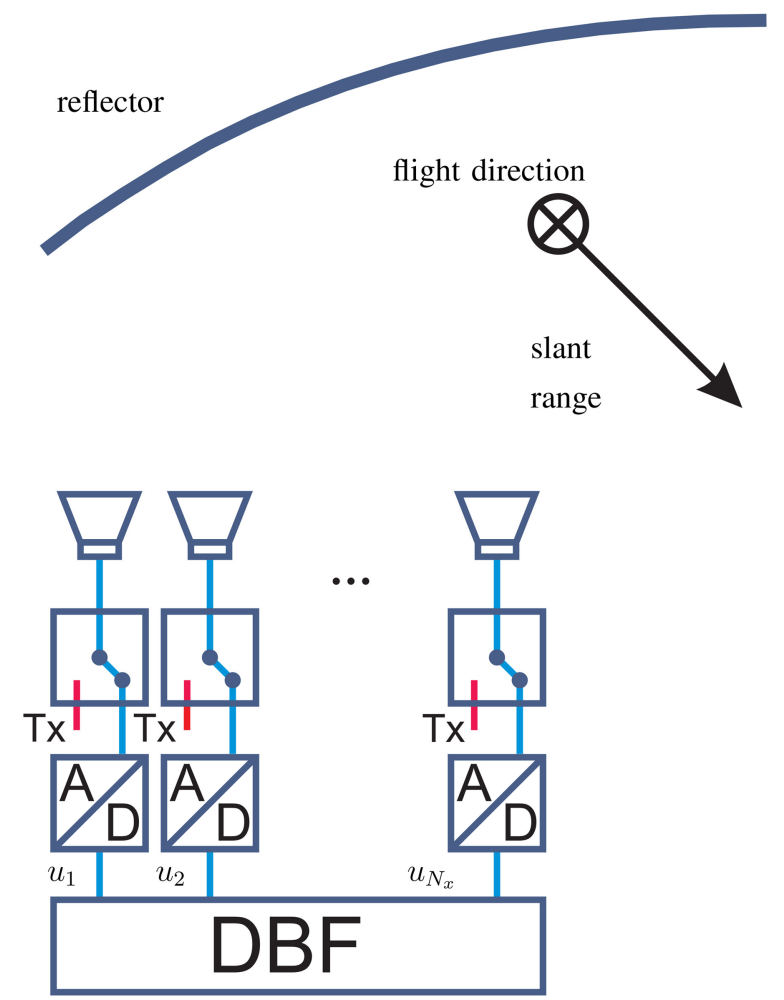

Fig. 1. Architecture for reflector system; some components such as LNAs, T/R modules, mixers, filters etc. are not shown to maintain clear representation.

shown. In order to facilitate DBF techniques in the azimuth dimension, with the goal of high resolution SAR imagery, the feed array is extended in both range and azimuth. This means that the feed array hardware is in principle repeated for every azimuth channel. The overall number of channels is then the number of elevation channels $N_{x}$ times the number of azimuth channels $N_{y}$.

\section{B. Reflector Antenna and Feed Array Design}

The antenna design presented in this article is for an X-band SAR system with a center frequency of $9.65 \mathrm{GHz}$ orbiting $785 \mathrm{~km}$ above the Earth surface. The swath width $X$ is $100 \mathrm{~km}$ starting at an incidence angle $\theta_{\mathrm{i}}$ of $30.5^{\circ}$ with an azimuth resolution $\Delta y$ better than $1 \mathrm{~m}$. The reflector design is of parabolic shape combined with a planar feed array with the parameters listed in Table I. The reflector patterns have been simulated using the reflector antenna analysis software TICRA GRASP9 [11]. Figure 2 shows the geometrical alignment of the reflector and the feed array. The diameter $D$ refers to the orthogonal projection of the reflector rim in the $x_{\mathrm{r}}^{\prime}-y_{\mathrm{r}}^{\prime}$-plane of the local reflector coordinate system $\left[x_{\mathrm{r}}^{\prime}, y_{\mathrm{r}}^{\prime}, z_{\mathrm{r}}^{\prime}\right]$. The focal length of 4.9 m results in an $F$ over $D$ ratio of 0.7 and the offset is the distance between the axes $z_{\mathrm{r}}^{\prime}$ and $z_{\mathrm{r}}$, where $z_{\mathrm{r}}$ is the rotational axis of the paraboloid. In contrast to the local antenna coordinate system $\left[x_{\mathrm{r}}^{\prime}, y_{\mathrm{r}}^{\prime}, z_{\mathrm{r}}^{\prime}\right]$ the coordinate system $\left[x_{\mathrm{r}}, y_{\mathrm{r}}, z_{\mathrm{r}}\right]$ only serves 

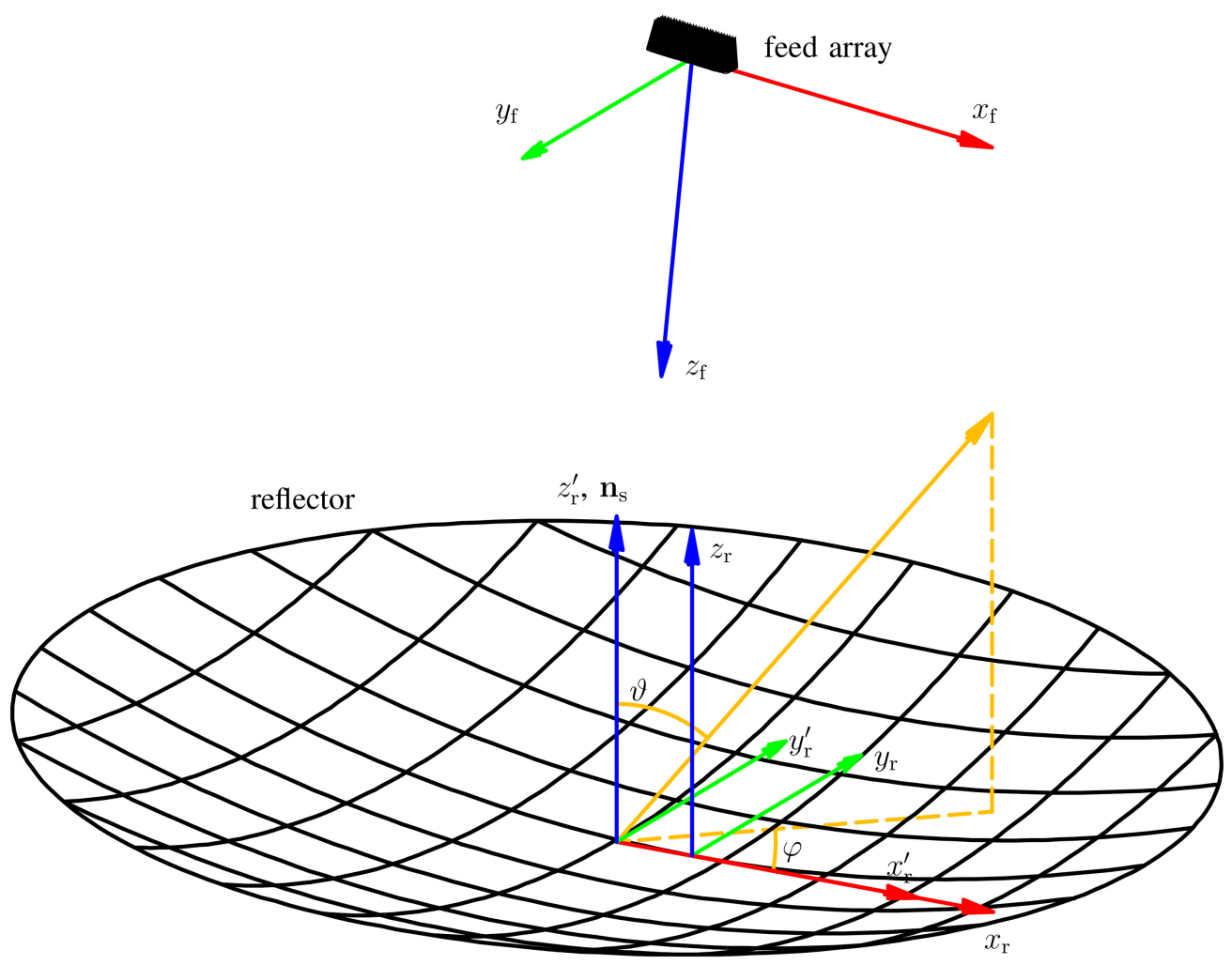

Fig. 2. Reflector and feed array geometry.

TABLE I

Reflector and Feed Design Parameters

\begin{tabular}{ccc}
\hline \hline Parameter & Symbol & Value \\
\hline diameter & $D$ & $7 \mathrm{~m}$ \\
focal length & $F$ & $4.9 \mathrm{~m}$ \\
offset & $O$ & $0.5 \mathrm{~m}$ \\
elevation feed element spacing & $\Delta x$ & $0.6 \lambda$ \\
azimuth feed element spacing & $\Delta y$ & $0.6 \lambda$ \\
no. of elevation feed elements & $N_{x}$ & 27 \\
no. of azimuth feed elements & $N_{y}$ & 6 \\
\hline
\end{tabular}

for the definition of the parabolic reflector with its minimum in the origin of this coordinate system. The element spacing within the planar feed array is in multiples of the wavelength $\lambda$ at the center frequency of $9.65 \mathrm{GHz}$. The feed element patterns are modeled as Gaussian beams with an edge taper of $-12 \mathrm{~dB}$. The feed array has an overall length in the $x_{\mathrm{f}}$-direction of approximately $0.5 \mathrm{~m}$ and a width in the azimuth direction of $0.1 \mathrm{~m}$ in the local feed coordinate system. In order to illuminate the reflector properly the feed array is tilted to the center of the local reflector coordinate system.

To cover the ground swath of $100 \mathrm{~km}$ the antenna beamwidth on transmit in elevation is approximately $5.2^{\circ}$ while the azimuth resolution requires an azimuth half power beamwidth of $0.89^{\circ}$. The polar gain pattern plot, where all feed elements are activated, is depicted in Fig. 3 showing a distinct rectangular shape.

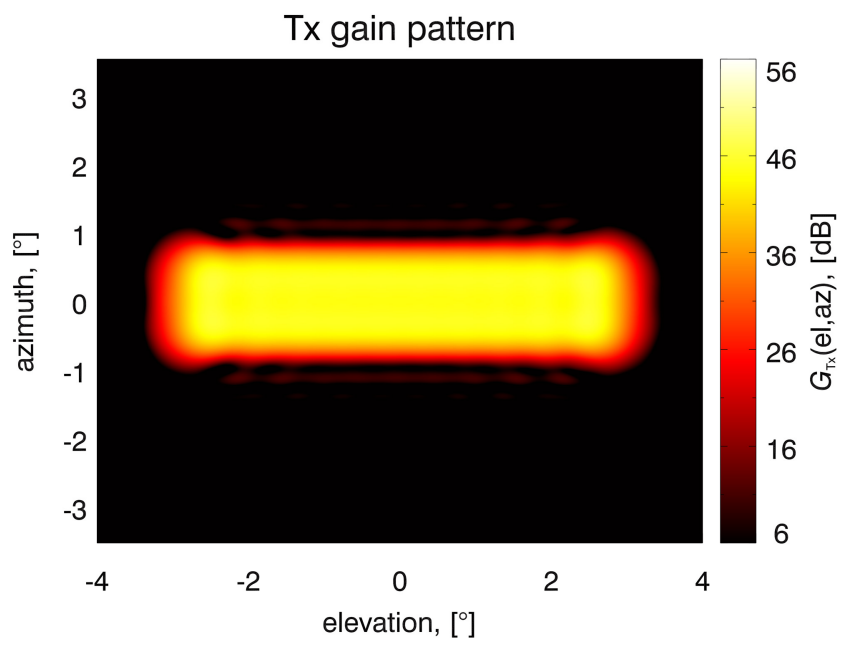

Fig. 3. Gain pattern on transmit.

As examples the gain patterns on receive for a center feed element and an off-focus element are presented in Fig. 4 and in Fig. 5, respectively. Clearly the off-focus feed element produces a broader pattern with slightly reduced gain compared with the center element. Additionally the sidelobe level increases, which results in a degraded azimuth performance. This can especially be observed in the corresponding cut plots in Fig. 6 and Fig. 7. An interesting effect can be observed in Fig. 6 on the most left pattern, where the sidelobes have an asymmetric level. These sidelobes are called coma lobes [12] and have been 


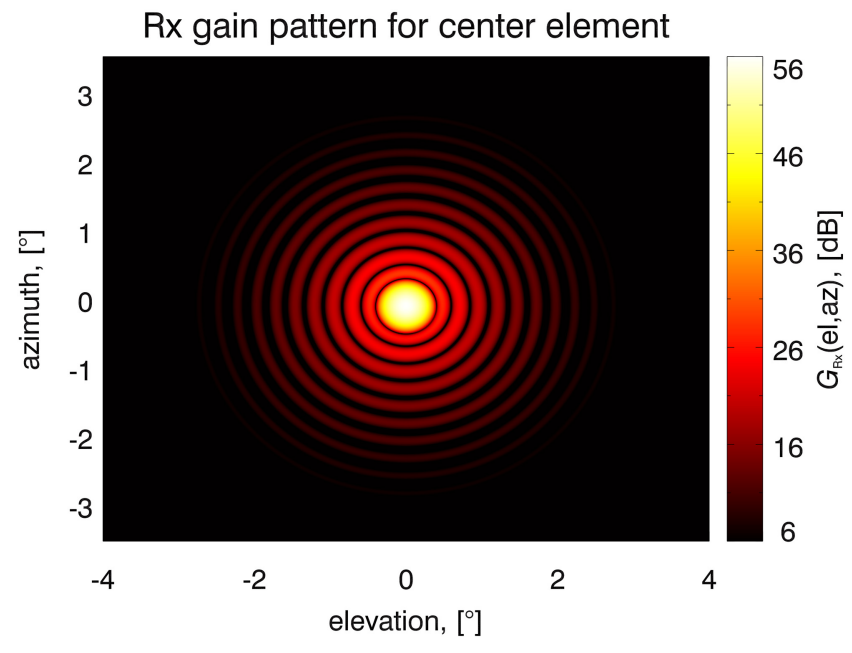

Fig. 4. Gain pattern on receive for center element.

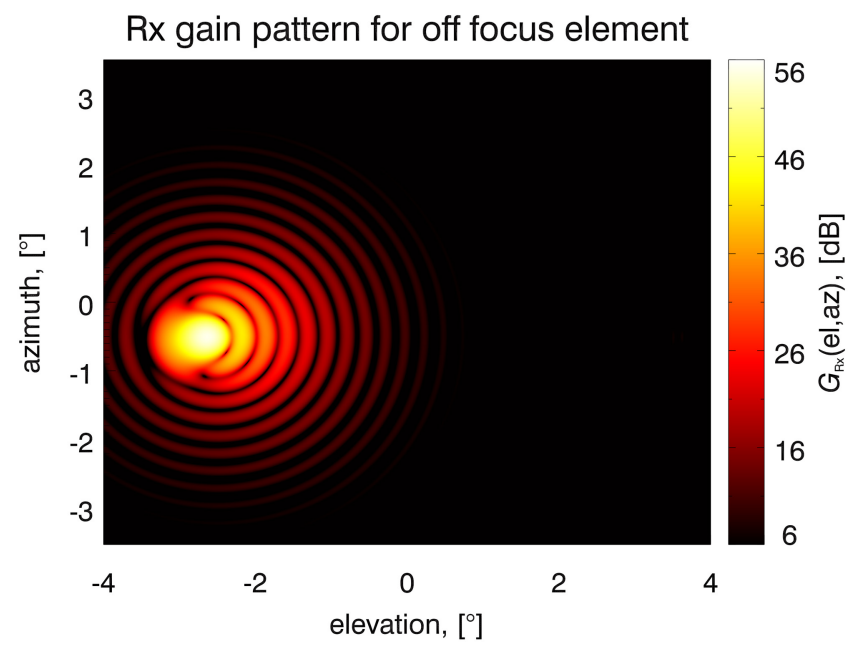

Fig. 5. Gain pattern on receive for off-focus element.

investigated for an off-focus reflector antenna for example in [13]. For radar systems without DBF coma lobes can be critical, because the system might become sensitive to spatial interference.

However, with DBF interferences can be suppressed. Interpreting the channel patterns as spatial filters, the typical bandpass character in the case of reflector antennas becomes obvious. This means every channel sees a different part of the spatial spectrum, which is in contrast to planar array antennas, where all channels see the same spectral domain. Another important feature of reflector antennas is the low peak-to-sidelobe ratio varying between $13 \mathrm{~dB}$ for the off-focus elements and $30.5 \mathrm{~dB}$ for the center elements.

\section{SYSTEM OPERATION}

On transmit, all feed elements are active in order to illuminate the complete swath as indicated by the yellow beam in Fig. 8. The emitted waveform is a chirp signal of duration $\tau_{\mathrm{p}}$. When the pulse hits the ground it moves from the near to the far end of the

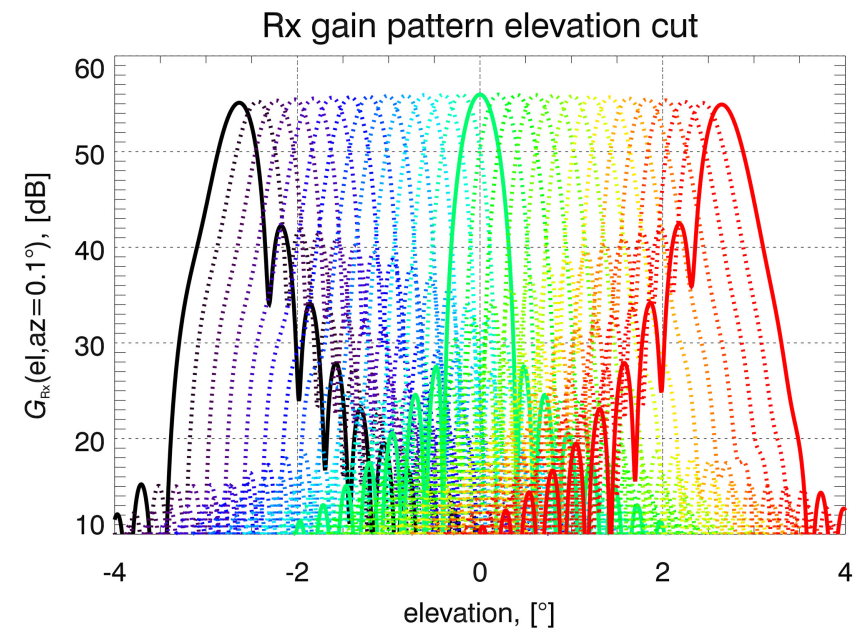

Fig. 6. Cut for 27 channel patterns in elevation.

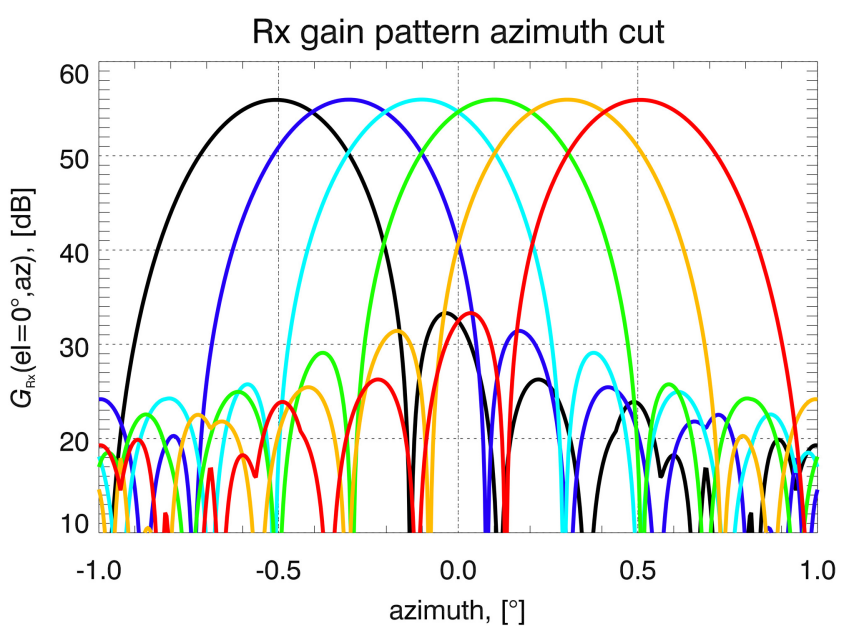

Fig. 7. Cut for 6 channel patterns in azimuth in center of array.

swath. This requires the receive beam to follow the echo on ground. This mode of operation is known as SCORE, first suggested by [14] and further developed by [15]-[17].

Since the chirp signal is a linearly modulated waveform, different spectral parts, symbolized by the rainbow colors in Fig. 8, will arrive at different time instances at the sensor. This peculiarity asks for more sophisticated beamforming techniques, where not only spatial DBF methods but also temporal beamforming approaches have to be taken into account. This is elaborated in detail in the next sections.

\section{DIGITAL BEAMFORMING CONCEPTS}

The next sections cover the digital signal processing strategies based upon a SAR signal model. The beamforming techniques are separated in elevation and azimuth.

\section{A. SAR Signal Model}

Essential for the following studies is the underlying physical SAR system model. SAR imaging 


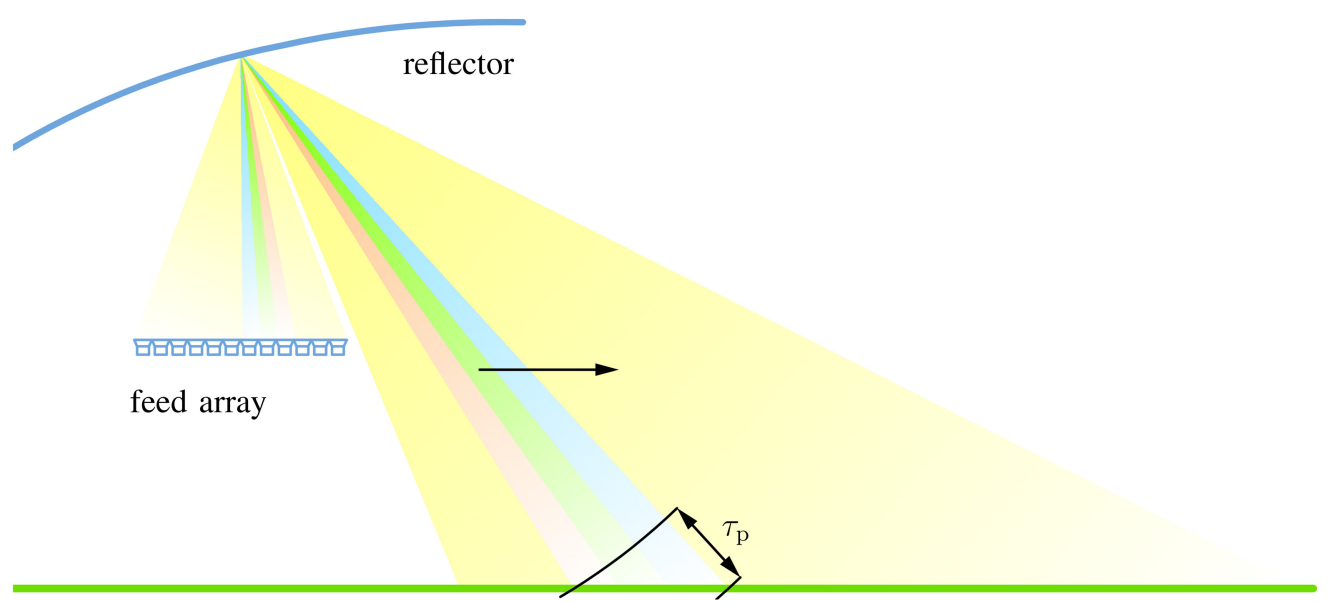

Fig. 8. System operation.

is an electromagnetic scattering problem which is covered by the Helmholtz equation. The solution based on Green's law in the context of SAR is known as imaging equation. This solution adapted to the SIMO problem for a monostatic setup for the $i$ th receive channel can be expressed according to (1). Here the received SAR raw data signal is denoted by $u_{i}$ as a function of the sensor location vector $\mathbf{r}_{\mathrm{s}}$, as indicated in Fig. 9, and time $t$. Commonly the sensor position variable is associated with azimuth and time with elevation or range. Within the first integrand on the right side $f$ is the target reflectivity as a function of the target location vector $\mathbf{r}=\left[\begin{array}{lll}x^{\prime} & y^{\prime} & z^{\prime}\end{array}\right]^{\mathrm{T}}$. $a_{i}$ denotes the complex two-way amplitude pattern as given in (2) as a function of the spherical variables $(\vartheta, \varphi)$ (see Fig. 2) in the local antenna coordinate system. $g$ given in (3) is the product of the transmitted signal of amplitude $p_{\mathrm{Tx}}$ and the Green's function describing the expansion of the waveform from the sensor location $\mathbf{r}_{\mathrm{s}}=\left[\begin{array}{lll}x & y & z\end{array}\right]^{\mathrm{T}}$ to the target and back to the receiver. Here $\tau$ is the delay time as defined in (7), $\tau_{\mathrm{p}}$ is the pulse duration, and $B$ denotes the signal bandwidth. The sum of integrals in (1), subscripted by $j$, describes the contributions of preceding and succeeding pulses which are known as range ambiguous returns. The raw signal is superimposed by bandlimited thermal receiver noise $v_{i}$.
Clearly the task of DBF in this paper is not the reconstruction of the SAR reflectivity function $f$. The DBF concepts derived here try to combine the raw data channels $u_{i}$ to a single output signal in a way that classical SAR focusing routines can be applied.

Principally all elevation and azimuth channels could be downlinked and processed on ground. Nevertheless the limiting factor is the data rate $R$, which can be written for a multi-channel system as

$$
R=N_{\text {chan }} \cdot 2 \cdot f_{\mathrm{s}} \cdot N_{\mathrm{b}} \cdot \mathrm{EWL} \cdot \mathrm{PRF}
$$

where $N_{\text {chan }}$ is the number of digital channels, $f_{\mathrm{s}}$ is the sampling frequency, $N_{\mathrm{b}}$ is the number of bits used for quantization and, EWL is the echo window length. The factor 2 accounts for in-phase and quadrature channel after complex demodulation.

Therefore it is crucial to perform as much of digital signal processing onboard the spacecraft as possible. The feed array architecture suggests a separation in elevation and azimuth. In order to avoid large onboard mass memories a good compromise is to perform DBF in elevation on board and to downlink the resulting azimuth channel signals.

\section{B. Digital Beamforming in Elevation}

For pulsed radar systems the time interval between two succeeding pulses is pulse repetition interval (PRI). Consequently all digital signal processing

$$
\begin{aligned}
u_{i}\left(\mathbf{r}_{\mathrm{s}}, t\right) & =\iiint_{\mathbf{r}} f(\mathbf{r}) a_{i}(\vartheta, \varphi) g\left(\mathbf{r}_{\mathrm{s}}, \mathbf{r}, t\right) \mathrm{d} \mathbf{r}+\sum_{\substack{j=-\infty \\
j \neq 0}}^{\infty} \iiint_{\mathbf{r}_{j}} f\left(\mathbf{r}_{j}\right) a_{i}\left(\vartheta_{j}, \varphi_{j}\right) g\left(\mathbf{r}_{\mathrm{s}}, \mathbf{r}_{j}, t\right) \mathrm{d} \mathbf{r}_{j}+v_{i}(t) \\
a_{i}(\vartheta, \varphi) & =g_{\mathrm{Tx}}(\vartheta, \varphi) g_{\mathrm{Rx}, i}(\vartheta, \varphi) \sqrt{\frac{\lambda^{2}}{4 \pi}} \\
g\left(\mathbf{r}_{\mathrm{s}}, \mathbf{r}, t\right) & =\underbrace{p_{\mathrm{Tx}} \operatorname{rect}\left(\frac{t-\tau-\tau_{\mathrm{p}} / 2}{\tau_{\mathrm{p}}}\right) \exp \left[\mathrm{j} \pi\left(B / \tau_{\mathrm{p}}\right)\left(t-\tau-\tau_{\mathrm{p}} / 2\right)^{2}\right]}_{\text {transmitted waveform }} \underbrace{\frac{e^{\mathrm{j} 2 \pi f_{\mathrm{c}}(t-\tau)}}{(4 \pi)^{2}\left\|\mathbf{r}_{\mathrm{s}}-\mathbf{r}\right\|^{2}}}_{\text {Green's function }} .
\end{aligned}
$$




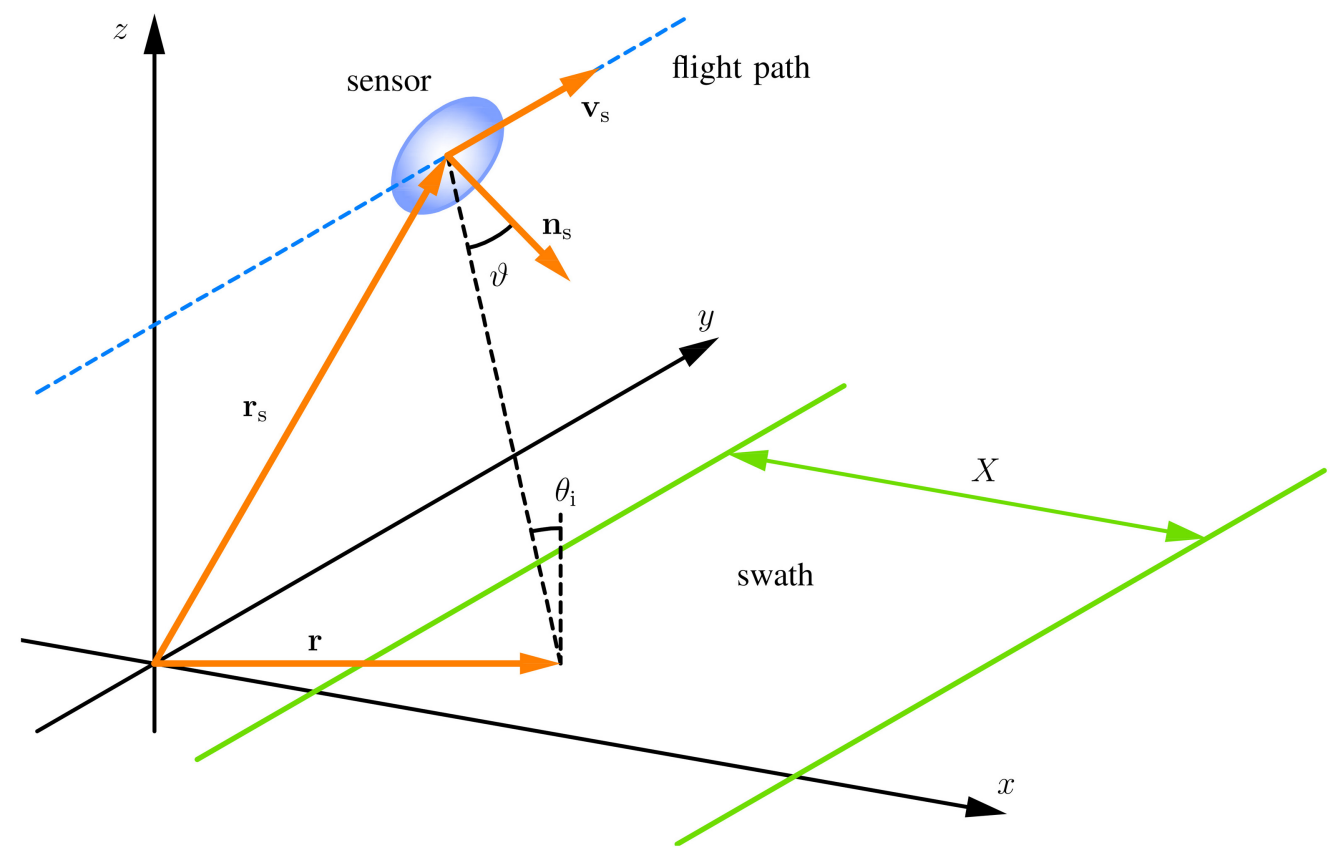

Fig. 9. Imaging geometry for reflector SAR system.

in elevation takes place in a time smaller than this interval. Even for simple beamforming algorithms the computational power required may exceed those of software based processors. Commonly for broadband applications field programmable gate arrays (FPGA) are used. In contrast to stationary systems, the filters to be implemented are time variant since the signal of interest varies its direction of arrival (DOA) over time as the pulse travels over ground (see Fig. 8). It is important to mention that the DOA for a specific target is constant. But since there are many targets making up the swath, the beamformer has to be adjusted to every target during one PRI. These time-variant filter coefficients can be read from a lookup table. The continuous time domain output of the digital beamformer at a fixed azimuth position can then be written as

$$
u_{\mathrm{DBF}}\left(\mathbf{r}_{\mathrm{s}}, t\right)=\sum_{i} \int_{t^{\prime}} u_{i}\left(\mathbf{r}_{\mathrm{s}}, t-t^{\prime}\right) h_{i}\left(t, t^{\prime}\right) \mathrm{d} t^{\prime}
$$

with $u_{i}\left(\mathbf{r}_{\mathrm{s}}, t\right)$ from (1). Important for the derivation of the filters $h_{i}$ is the fact that there exists a unique functional relationship between the target reflectivity coordinates $\mathbf{r}$, the antenna angle $\vartheta$, and the delay time $\tau$. Note, here $\vartheta$ refers to elevation in the context of elevation beamforming $\left(\varphi=0^{\circ}\right)$. The target coordinates are related to the antenna angle according to

$$
\cos \vartheta=\frac{\mathbf{n}_{\mathrm{s}}^{\mathrm{T}}\left(\mathbf{r}-\mathbf{r}_{\mathrm{s}}\right)}{\left\|\mathbf{n}_{\mathrm{s}}\right\| \cdot\left\|\mathbf{r}-\mathbf{r}_{\mathrm{s}}\right\|}
$$

with $\|$.$\| the 2-norm. Here the antenna normal$ vector $\mathbf{n}_{\mathrm{s}}$ is associated with the $z_{\mathrm{r}}^{\prime}$-axis of the local reflector coordinate system (see Fig. 2). Here the target position $\mathbf{r}$ is assumed to be known, since the problem of DOA estimation is out of the scope of this paper. In practice the knowledge of the orbit as well as a coarse digital elevation model (DEM) would be helpful or even required. The impact of imprecise DOA knowledge has been analyzed in [6] and adaptive DBF techniques in the frame of SAR have been studied in [18]. The delay time $\tau$ is related to the target coordinates $\mathbf{r}$ via

$$
\tau=2\left\|\mathbf{r}_{\mathrm{s}}-\mathbf{r}\right\| / c
$$

with $c$ the velocity of light. Even more important for the filter design is the characteristic of the transmitted waveform. Using a chirp signal the instantaneous frequency $f$ depends linearly on the time $t$

$$
f=\left(B / \tau_{\mathrm{p}}\right)\left(t-\tau-\tau_{\mathrm{p}} / 2\right)+f_{\mathrm{c}} .
$$

The reason why temporal beamforming is required can be clarified with Fig. 10. Consider two point targets received at different time instances, but partially overlapping in time domain, in the $i$ th elevation channel. Consequently these two point targets are also seen under different aspect angles and therefore weighted with different parts of the pattern. Clearly the problem is now that at any time instance in the temporal overlap the beamforming can only match one point target or the other. A possible solution to discriminate the two targets is to expand the signal into the time-frequency domain as shown in Fig. 10. Now the two targets can be weighted properly in the beamforming process.

With the above identities the basic idea of the filtering approaches presented here is to decompose the received signal into multiple frequency bands and to apply a specific filter on each of these individual 


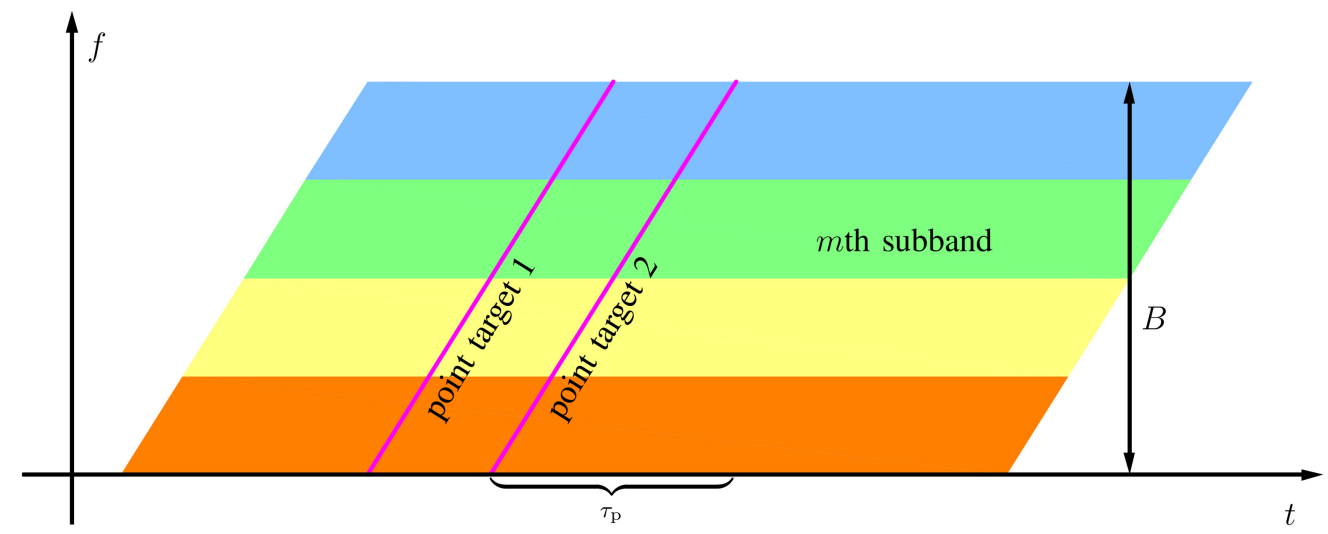

Fig. 10. Time-frequency domain representation of $i$ th raw data signal divided into subbands.

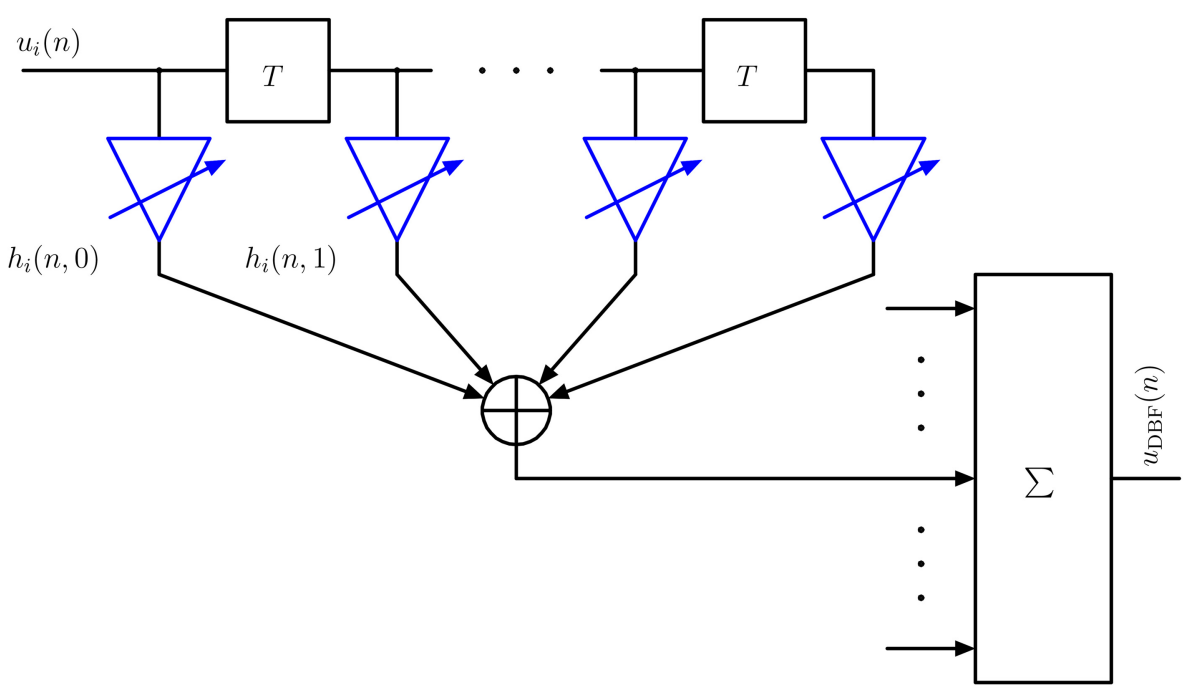

Fig. 11. FIR filter with time-variant coefficients.

subbands as sketched in Fig. 10. To each subband a bandpass filter (see (15)) and a weight is applied which is derived from its corresponding channel pattern. These individual bandpass filters are then combined to a single filter (see (10)) as presented in Fig. 11.

The time discrete $(t \rightarrow n)$ beamformer output, dropping the azimuth position $\mathbf{r}_{\mathrm{s}}$, writes

$$
u_{\mathrm{DBF}}(n)=\sum_{i \in Z_{\text {act }}(n)} \sum_{n^{\prime}=0}^{N_{\text {coef }}-1} u_{i}\left(n-n^{\prime}\right) h_{i}\left(n, n^{\prime}\right)
$$

with

$$
h_{i}\left(n, n^{\prime}\right)=\sum_{m=0}^{M-1} w_{i}(n, m) \bar{h}\left(n^{\prime}, m\right) .
$$

$Z_{\text {act }}(n)$ is the set of activated channels. Typically, this set comprises neighboring feed elements. $N_{\text {coef }}$ is the number of filter coefficients for a finite impulse response (FIR) filter of order $N_{\text {coef }}-1$ and $M$ is the number of subbands. Figure 11 shows a block diagram of such a filter with delay elements denoted by the clock period $T=1 / f_{\mathrm{s}}$. The filter design for $\bar{h}$ is based on a Fourier analysis of a bandpass transfer function of rectangular shape with cutoff frequencies $\Omega_{\mathrm{c} 1}$ and $\Omega_{\mathrm{c} 2}$. Then the Fourier series coefficients are

$$
\bar{h}(n, m)=\frac{1}{2 \pi} \int_{\Omega_{\mathrm{c} 1}}^{\Omega_{\mathrm{c} 2}} e^{\mathrm{j} n \Omega} \mathrm{d} \Omega, \quad \Omega=2 \pi \frac{f}{f_{\mathrm{s}}}
$$

resulting in

$$
\begin{aligned}
\bar{h}(n, m)= & \frac{\Omega_{\mathrm{c} 2}-\Omega_{\mathrm{c} 1}}{2 \pi} \cdot \operatorname{sinc}\left(n \frac{\Omega_{\mathrm{c} 2}-\Omega_{\mathrm{c} 1}}{2}\right) \\
& \times \exp \left[\frac{\mathrm{j} n\left(\Omega_{\mathrm{c} 1}+\Omega_{\mathrm{c} 2}\right)}{2}\right] .
\end{aligned}
$$

Dividing the design bandwidth $\bar{B}$ into $M$ subbands of equal width, the cutoff frequencies are given as

$$
\begin{aligned}
& \Omega_{\mathrm{c} 1}=2 \pi\left(\frac{m}{M}-\frac{1}{2}\right) \frac{\bar{B}}{f_{\mathrm{s}}}, \\
& m \in[0, M-1] \\
& \Omega_{\mathrm{c} 2}=2 \pi\left(\frac{m+1}{M}-\frac{1}{2}\right) \frac{\bar{B}}{f_{\mathrm{s}}} \\
& m \in[0, M-1] .
\end{aligned}
$$


FIR filters of 30th order

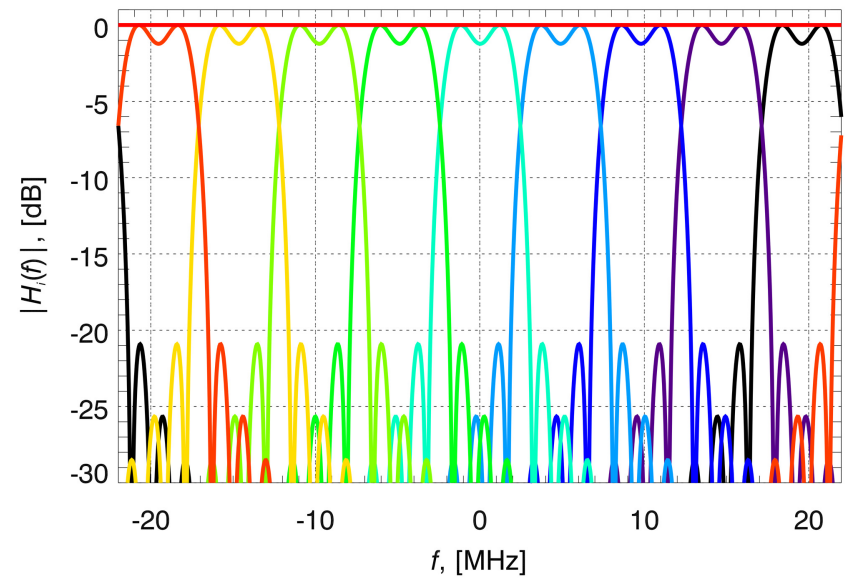

Fig. 12. FIR filter bank with nine subbands and after ideal reconstruction (constant curve).

The resulting filter coefficients equate

$$
\begin{aligned}
\bar{h}(n, m)= & \frac{\bar{B}}{M f_{\mathrm{s}}} \cdot \operatorname{sinc}\left(n \pi \frac{\bar{B}}{M f_{\mathrm{s}}}\right) \\
& \times \exp \left[\frac{\mathrm{j} n \pi((2 m+1) / M-1) \bar{B}}{f_{\mathrm{s}}}\right] .
\end{aligned}
$$

For the filter bank design it has to be ensured that the signal after combining the single filter outputs reconstructs the input signal. From (10) with the above definitions it can be shown that

$$
\sum_{m=0}^{M-1} w_{i}(n, m) \bar{h}(n, m)=\frac{\bar{B}}{f_{\mathrm{s}}} \operatorname{sinc}\left(n \pi \frac{\bar{B}}{f_{\mathrm{s}}}\right)
$$

assuming unity weights $w_{i}(n, m)$. In order to avoid aliasing, the sampling frequency $f_{\mathrm{s}}$ must be equal or larger than the signal bandwidth $B$. Consequently it would be sufficient to restrict the design bandwidth $\bar{B}$ to the signal bandwidth. However, setting $\bar{B}$ to $f_{\mathrm{s}}$ offers

$$
\frac{\bar{B}}{f_{\mathrm{s}}} \operatorname{sinc}\left(n \pi \frac{\bar{B}}{f_{\mathrm{s}}}\right)=\operatorname{sinc}(n \pi)=\delta(n) .
$$

From this result it can be concluded that the reconstruction is ideal and independent from the number of filter coefficients $N_{\text {coef }}$ for $w_{i}(n, m)=1$. Figure 12 shows the transfer functions for a filter bank with nine subbands and 31 coefficients, which are used in the performance analysis in Section V-A. The constant curve represents the transfer function after ideal reconstruction given by (17). The low signal bandwidth of $40 \mathrm{MHz}$ has been chosen in order to reduce the computational load.

Due to the linear time-frequency relationship of chirp signals every subspectrum enters a feed element at a defined time for a given direction. Since the chirp spectrum is divided into $M$ subbands the time-dependent weights are taken at the center of each subspectrum. Introducing the subband specific delay $n_{m}$ the weights in (10) can be rewritten as

$$
w_{i}(n, m)=w_{i}\left(n-n_{m}\right)
$$

with

$$
n_{m}=\operatorname{nint}\left\{\frac{2 m+1}{2 M} \tau_{\mathrm{p}} f_{\mathrm{s}}\right\}
$$

with nint $\{$.$\} the nearest integer function.$

Another important issue, which is only briefly addressed, has been analyzed in [19] for an L-band reflector antenna. The patterns show a strong dependency on the transmitted frequency. That means the signal is modulated over the frequency band of interest. This can be accounted for in the time-variant beamforming approach, simply by using the corresponding patterns for the individual subbands. The weights $w_{i}(n, m)$ would then additionally become a function of the frequency $w_{i}(n, m, f)$. However, in the frame of the following investigations the patterns are assumed to be constant over the frequency band under consideration.

Having derived the time-variant filters, in the following the most important performance figures for different beamforming approaches are discussed. The fundamental performance parameter for any radar system is the signal-to-noise ratio (SNR). Assuming zero mean circular complex Gaussian random processes for the backscatter as well as for the noise, the discrete time-dependent expression for the SNR can be shown to be (see the Appendix)

$$
\operatorname{SNR}(n) \approx \frac{\left|\sum_{n}^{n+N_{\mathrm{p}}} \sqrt{P_{s}(n)}\right|^{2}}{\sum_{n}^{n+N_{\mathrm{p}}} P_{v}(n)}
$$

with the signal power

$$
P_{s}(n)=\sigma_{f}^{2}(\vartheta)\left|\mathbf{a}^{\mathrm{T}}(\vartheta) \sum_{n^{\prime}} g\left(\vartheta, n-n^{\prime}\right) \mathbf{h}\left(n, n^{\prime}\right)\right|^{2}
$$

and the noise power

$$
P_{v}(n)=\sum_{n^{\prime}} \mathbf{h}^{\mathrm{T}}\left(n, n^{\prime}\right) \mathbf{R}_{\mathbf{v}}\left(n-n^{\prime}\right) \mathbf{h}^{*}\left(n, n^{\prime}\right)
$$

where $(.)^{*}$ means conjugate complex. Here $N_{\mathrm{p}}=$ $\operatorname{nint}\left\{\tau_{\mathrm{p}} \cdot f_{\mathrm{s}}\right\}$ is the number of samples per pulse. The backscatter radar cross section represented by $\sigma_{f}^{2}$ is the product of the backscatter coefficient $\sigma_{0}$ and the resolution cell $A_{\text {cell }} . \mathbf{a}(\vartheta)=\left[a_{1}(\vartheta) a_{2}(\vartheta) \cdots a_{N_{x}}(\vartheta)\right]^{\mathrm{T}}$ is the antenna steering vector, which contains the individual complex element patterns. The term $g\left(\vartheta, n-n^{\prime}\right)$ uses (6) to substitute $\mathbf{r}_{\mathrm{s}}$ and $\mathbf{r}$ (compare with left side of (3)). Important to mention is that in this SNR formula the so-called azimuth compression gain is not taken into account. Nevertheless (20) serves as basis for the comparison of different beamformers which are presented in the following.

The most rudimental beamforming approach is simply to turn on those channels which receive signal 
power from the pulse traveling over ground. In the reflector case this implies that only a subset of feed elements $N_{\text {act }}$ is activated at a time instance. For a single band filter $(M=1)$ the filter impulse response vector $\mathbf{h}\left(n, n^{\prime}\right)$ is

$$
\mathbf{h}\left(n, n^{\prime}\right)=\mathbf{w}(n) \delta\left(n^{\prime}\right) .
$$

The weight vector $\mathbf{w}$ at a time instance $n$ would, for example, look like this:

$$
\mathbf{w}(n)=\left[\begin{array}{llllll}
0 & 1 & 1 & 1 & 0 & \cdots
\end{array}\right]^{\mathrm{T}}
$$

where the number of activated elements is three in this example. The corresponding SNR analogous to (20) is then

$$
\operatorname{SNR}(n)=\frac{\left|\sum_{N_{\mathrm{p}}} \sqrt{\sigma_{f}^{2}(\vartheta)|g(\vartheta, n)|^{2}\left|\mathbf{a}^{\mathrm{T}}(\vartheta) \mathbf{w}(n)\right|^{2}}\right|^{2}}{\sum_{N_{\mathrm{p}}} \mathbf{w}^{\mathrm{T}}(n) \mathbf{R}_{\mathbf{v}}(n) \mathbf{w}^{*}(n)} .
$$

Assuming $\mathbf{R}_{\mathbf{v}}(n)=\sigma_{v}^{2}(n) \mathbf{I}$, with $\mathbf{I}$ the identity matrix, it becomes clear that the SNR for this beamformer drops proportional to the number of activated channels $N_{\text {act }}$.

$$
\operatorname{SNR}(n)=\frac{\left|\sum_{N_{\mathrm{p}}} \sqrt{\sigma_{f}^{2}(\vartheta)|g(\vartheta, n)|^{2}\left|\mathbf{a}^{\mathrm{T}}(\vartheta) \mathbf{w}(n)\right|^{2}}\right|^{2}}{\sum_{N_{\mathrm{p}}} \sigma_{v}^{2}(n) N_{\mathrm{act}}} .
$$

This is due to the fact that most channels contribute only with noise on receive. Nevertheless this beamformer is justified by the fact that it is technically easy and cost effective to implement. Moreover this beamformer is robust in terms of coefficient stability, because no filter design incorporating knowledge of the complex amplitude antenna pattern is required. Beamformer (24) is denoted as unity beamformer throughout this article. In the following the variables $\vartheta$ and $n$ are dropped for notational simplicity.

A second class of beamformers is known as minimum variance distortionless response (MVDR) beamformer [20] which is based on Capon's method [21]. This method preserves the signal of interest while minimizing contributions to the beamformer output due to interference from other directions than the direction of interest and noise. This approach can be understood as a spatial matched filter. It is optimal with respect to the SNR. The cost function of minimizing the variance of the beamformer output subject to the constraint writes

$$
\begin{array}{ll}
\text { minimize } & \mathbf{w}^{\mathrm{T}} \mathbf{R}_{\mathbf{u}} \mathbf{w}^{*} \\
\text { subject to } & \mathbf{a}^{\mathrm{T}} \mathbf{w}=1 .
\end{array}
$$

Since the noise covariance matrix $\mathbf{R}_{\mathrm{v}}$ is typically unknown it is replaced by the channel covariance matrix $\mathbf{R}_{\mathbf{u}}$. The optimum conjugate complex weight vector $\mathbf{W}^{*}$ in closed form is given as

$$
\mathbf{w}^{*}=\frac{\mathbf{R}_{\mathbf{u}}^{-1} \mathbf{a}}{\mathbf{a}^{\mathrm{H}} \mathbf{R}_{\mathbf{u}}^{-1} \mathbf{a}}
$$

with $\mathbf{R}_{\mathbf{u}}$ to be estimated from $N$ recently received samples.

$$
\mathbf{R}_{\mathbf{u}}(n)=\frac{1}{N} \sum_{n^{\prime}=n-N+1}^{n} \mathbf{u}\left(n^{\prime}\right) \mathbf{u}^{\mathrm{H}}\left(n^{\prime}\right) \in \mathbb{C}^{N_{\text {act }} \times N_{\text {act }}}
$$

where $(.)^{\mathrm{H}}$ means conjugate transpose (Hermitian). Again for a single band filter $(M=1)$ and $\mathbf{R}_{\mathbf{u}}$ assumed to be $\sigma_{u}^{2} \mathbf{I}$ and $\mathbf{R}_{\mathbf{v}}=\sigma_{v}^{2} \mathbf{I}$ the SNR for the MVDR beamformer according to (20) yields

$$
\mathrm{SNR}=\frac{\left|\sum_{N_{\mathrm{p}}} \sqrt{\sigma_{f}^{2}|g|^{2}}\right|^{2}}{\sum_{N_{\mathrm{p}}} \sigma_{v}^{2}\left(\mathbf{a}^{\mathrm{T}} \mathbf{a}^{*}\right)^{-1}}
$$

In principle the MVDR beamformer allows to activate all channels on receive simultaneously. Those channels contributing predominantly with noise are quasi nulled with small magnitude weights. Since the signals are also combined according to their phase, the high receive gain can be reconstructed at every time instance. However it is advisable to restrict the activated feed elements to those where the complex amplitude pattern is accurately known. Otherwise the spatial filter is mismatched and the SNR will degrade slightly. This also applies to the beamformer presented in the following.

Pulsed SAR systems generally suffer from preceding and succeeding pulse echoes arriving at the same time at the sensor as the signal of interest. Those echoes are called range ambiguities since they enter the system under different elevation angles. One possibility to suppress those ambiguous directions is by means of a spatial filter. A method known as linear constraint minimum variance (LCMV) beamforming [20] provides an analytic solution to this problem. The problem is stated as follows

$$
\begin{aligned}
\operatorname{minimize} & \mathbf{w}^{\mathrm{T}} \mathbf{R}_{\mathbf{u}} \mathbf{w}^{*} \\
\text { subject to } & \mathbf{A}^{\mathrm{T}} \mathbf{w}=\mathbf{c}
\end{aligned}
$$

with the array response matrix

$$
\mathbf{A}=\left[\begin{array}{l}
\mathbf{a}(\vartheta) \cdots \mathbf{a}\left(\vartheta_{-1}\right) \\
\mathbf{a}\left(\vartheta_{1}\right) \cdots
\end{array}\right] \in \mathbb{C}^{N_{\text {act }} \times N_{\text {dir }}}
$$

and the constraint vector $\mathbf{c}=\left[\begin{array}{lll}1 & 0 & \cdots\end{array}\right]^{\mathrm{T}} \in \mathbb{C}^{N_{\mathrm{act}} \times 1} . N_{\mathrm{dir}}$ denotes the number of directions under consideration. The closed-form solution is

$$
\mathbf{w}^{*}=\mathbf{R}_{\mathbf{u}}^{-1} \mathbf{A}\left(\mathbf{A}^{\mathrm{H}} \mathbf{R}_{\mathbf{u}}^{-1} \mathbf{A}\right)^{-1} \mathbf{c} .
$$

Equation (35) represents the least squares solution to a quadratic optimization problem. The key step in the numerical evaluation of (35) is the computation of the inverse of $\mathbf{A}^{\mathrm{H}} \mathbf{R}_{\mathbf{u}}^{-1} \mathbf{A}$. Even if the channels are well balanced $\left(\mathbf{R}_{\mathbf{u}}^{-1} \sim \mathbf{I}\right)$ and the number of directions to be suppressed is lower than the number of channels, this matrix can become ill posed quickly. This is because of the strong focussing effect of the reflector pattern where every channel illuminates a different solid angle. That means that one or more directions 
only contribute with small magnitudes and the matrix becomes rank deficient. A possible way to solve such an inversion problem is by means of eigenvalue thresholding techniques [22]. In analogy to the previous beamformers the SNR can be computed using (25) and (35).

The performance figure measuring the amount of ambiguous signal power superimposing the signal of interest is the range-ambiguity-to-signal ratio (RASR). This quotient can be expressed for a point target according to

$$
\operatorname{RASR}(n)=\frac{P_{a}(n)}{P_{s}(n)}
$$

with the signal power $P_{s}(n)$

$$
P_{s}(n)=\sigma_{f}^{2}(\mathbf{r}) \frac{\left|\mathbf{a}^{\mathrm{T}}(\vartheta) \mathbf{w}(n)\right|^{2}}{\left\|\mathbf{r}_{\mathrm{s}}-\mathbf{r}\right\|^{3} \sin \theta_{\mathrm{i}}}
$$

and the ambiguous power $P_{a}(n)$

$$
P_{a}(n)=\sum_{\substack{j=-\infty \\ j \neq 0}}^{\infty} \sigma_{f}^{2}\left(\mathbf{r}_{j}\right) \frac{\left|\mathbf{a}^{\mathrm{T}}\left(\vartheta_{j}\right) \mathbf{w}(n)\right|^{2}}{\left\|\mathbf{r}_{\mathrm{s}}-\mathbf{r}_{j}\right\|^{3} \sin \theta_{\mathrm{i}, j}} .
$$

The distance from the sensor to the ambiguous regions $\mathbf{r}_{j}$ is

$$
\left\|\mathbf{r}_{\mathrm{s}}-\mathbf{r}_{j}\right\|=\left\|\mathbf{r}_{\mathrm{s}}-\mathbf{r}\right\|+j \frac{c}{2 \mathrm{PRF}} .
$$

\section{Digital Beamforming in Azimuth}

Conventional pulsed SAR systems, specifically single-channel systems, are inherently restricted with respect to their imaging capability. With these systems it is not possible to achieve a large swath width and a high azimuth resolution at the same time [23]. A high resolution requires a broad beam, which needs a large PRF in order to sample adequately (Nyquist). The high PRF in turn limits the swath width. The swath width $X$ and the azimuth resolution $\Delta y$ can be shown to be related [24] according to

$$
\frac{X}{\Delta y} \leq \frac{c}{2 v \sin \theta_{\mathrm{i}}}
$$

where $c$ and $v$ are the velocities of light and the sensor, respectively, and $\theta_{\mathrm{i}}$ is the incidence angle (see Fig. 9). This quotient only depends on physical constants except for the imaging geometry.

One possibility to overcome this restriction is to transmit a signal using a broad beam and to collect the scattered signal with multiple receivers. The individual signals are then processed in order to reconstruct the high resolution image. A simple way to implement such a system using a reflector antenna is to extend the digital feed array in the azimuth dimension $[4,5]$.

DBF in azimuth principally applies in the same way as it does in elevation. A set of channels in azimuth is used to reconstruct a single high gain signal subject to certain constraints.
The underlying signal model is again based on (1), (2), and (3), except that the time dependence is ignored and the equations are evaluated over the spatial azimuth variable $y$, which is contained in the sensor flight trajectory $\mathbf{r}_{\mathrm{s}}(y)$. Equation (3) is then simply

$$
g\left(\mathbf{r}_{\mathrm{s}}(y)\right)=\frac{e^{-\mathrm{j} 2 \pi f_{\mathrm{c}} \tau}}{(4 \pi)^{2}\left\|\mathbf{r}_{\mathrm{s}}-\mathbf{r}\right\|^{2}} .
$$

The spherical antenna coordinate $\vartheta$ now refers to the azimuthal plane $\left(\varphi=90^{\circ}\right)$ (see Fig. 2).

A compact way to relate space, represented by $\vartheta$, and Doppler frequency $f_{y}$ is by means of wavenumbers. The azimuth wavenumber $k_{y}$ can be written for a monostatic setup and a linear flight trajectory as

$$
k_{y}=-2 k \sin \vartheta, \quad k=\frac{2 \pi}{\lambda}
$$

with $\lambda$ the wavelength. The Doppler frequency $f_{y}$ and the PRF are related to the Doppler wavenumber $k_{y}$ and the sampling wavenumber $K_{y}$, respectively, according to

$$
k_{y}=2 \pi \frac{f_{y}}{v}, \quad K_{y}=2 \pi \frac{\mathrm{PRF}}{v} .
$$

The DBF approach presented here is based on a wavenumber analysis of the azimuth signal. That means any DBF algorithms are applied in the wavenumber domain. The $i$ th azimuth channel raw signal in the continuous wavenumber domain writes

$$
U_{i}\left(k_{y}\right)=\frac{1}{v} \int_{-\infty}^{\infty} u_{i}(y) e^{-\mathrm{j} k_{y} y} \mathrm{~d} y .
$$

Since the azimuth signal is naturally a discrete signal, sampled with $K_{y}$ or PRF, respectively, the problem of aliasing arises. As stated before it is the aim to achieve a high azimuth resolution and a large swath width. Since the data rate imposes a stringent constraint, $K_{y}$ has to be chosen as low as possible. However, the minimum possible $K_{y}$ is determined by what is called in [2] the diffraction limit. This means that in the DBF process a minimum beamwidth can be reconstructed which is determined by the physical length of the antenna. For small azimuth half power beamwidths $\vartheta_{3 \mathrm{~dB}} \approx 1.22 \lambda / D$ [12] the lower bound for $K_{y}$ can be shown to be

$$
K_{y} \geq 4 k \sin \left(\frac{\vartheta_{3 \mathrm{~dB}}}{2}\right) \approx 1.22 \frac{4 \pi}{D}
$$

with $D$ the reflector antenna diameter. This means that every azimuth channel is undersampled and therefore subject to aliasing. The azimuth processing approach can therefore be characterized as a two-stage process. First, in order to form the high resolution image, the individual azimuth channels have to be reconstructed on a common grid with a high sampling rate $K_{y}^{\prime}$. And secondly beamforming concepts have to be applied to suppress the aliased azimuth signal energy. The 


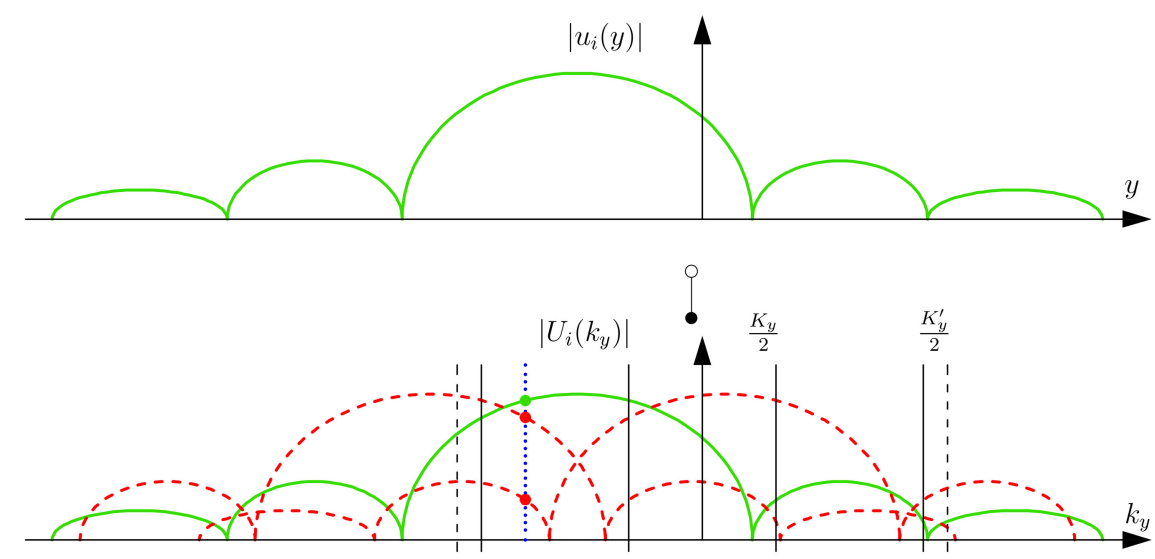

Fig. 13. Wavenumber domain representation of sampled signal.

approach presented here is based on the discrete version of (44) over a finite domain

$$
\begin{aligned}
& U_{i}\left(m \Delta k_{y}\right) \\
& \quad=\frac{\Delta y}{v} \sum_{n=0}^{N-1} u_{i}(y(n \Delta y)) \exp \left[-\mathrm{j} k_{y}\left(m \Delta k_{y}\right) y(n \Delta y)\right]
\end{aligned}
$$

with the wavenumber sampling increment $\Delta k_{y}$

$$
\Delta k_{y}=\frac{2 \pi}{Y}=\frac{2 \pi}{M \Delta y^{\prime}}=\frac{K_{y}^{\prime}}{M}, \quad k_{y} \in\left[-\frac{K_{y}^{\prime}}{2}, \frac{K_{y}^{\prime}}{2}\right]
$$

and

$$
N=\frac{Y}{\Delta y}, \quad M=\frac{Y}{\Delta y^{\prime}} .
$$

Here $Y$ is the complete integration path length. $N$ is the number of samples of the undersampled channel signals and $M$ is the number of samples of the high resolution signal. $\Delta y$ and $\Delta y^{\prime}$ are the corresponding spatial sampling increments. Figure 13 illustrates the evaluation of (46). In the upper part the magnitude of the $i$ th azimuth signal $u_{i}(y)$ is depicted. The shape is due to the two-way channel pattern described by (2). By taking the discrete Fourier transform according to (46) not only the principal interval, restricted to $\pm K_{y} / 2$, becomes visible but also additional replicas of the spectrum beyond the baseband as can be observed in the lower part of Fig. 13. Here the first left and first right ambiguous spectrum is plotted (dashed curves). In fact (46) is evaluated over the desired wavenumber domain $\pm K_{y}^{\prime} / 2$. Evidently this procedure can be implemented in a computationally more efficient way by using the fast Fourier transform (FFT), where the spectra are juxtaposed and limited to the bandwidth $K_{y}^{\prime}$. From an information theoretical point of view it is enough to just use the principal Doppler band $K_{y}$, because no new information is generated.

The second step in the azimuth processing is the combination of the individual channel spectra $U_{i}\left(k_{y}\right)$ by means of weights

$$
U_{\mathrm{DBF}}\left(k_{y}\right)=\mathbf{w}^{\mathrm{T}}\left(k_{y}\right) \mathbf{U}\left(k_{y}\right) \text {. }
$$

Again an SNR expression as function of the azimuth variable $y^{\prime}$ for a point target similar to (20) can be derived as

$$
\operatorname{SNR}\left(y^{\prime}\right)=\frac{\left|\int_{K_{y}^{\prime}}\right| \mathbf{w}^{\mathrm{T}}\left(k_{y}\right) \mathbf{s}\left(y^{\prime}, k_{y}\right)\left|\mathrm{d} k_{y}\right|^{2}}{\int_{K_{y}^{\prime}} \mathbf{w}^{\mathrm{T}}\left(k_{y}\right) \mathbf{R}_{\mathbf{v}}\left(k_{y}\right) \mathbf{w}^{*}\left(k_{y}\right) \mathrm{d} k_{y}}
$$

with the point target signal spectrum

$$
\begin{aligned}
\mathbf{s}\left(y^{\prime}, k_{y}\right) & =\frac{1}{v} \int_{-\infty}^{\infty} f\left(y^{\prime}\right) \mathbf{a}(\vartheta) g\left(y-y^{\prime}\right) e^{-\mathrm{j} k_{y} y} \mathrm{~d} y \\
& \approx \frac{1}{v} f\left(y^{\prime}\right) \mathbf{a}(\vartheta) \int_{-\infty}^{\infty} g\left(y-y^{\prime}\right) e^{-\mathrm{j} k_{y} y} \mathrm{~d} y .
\end{aligned}
$$

Here an azimuth compression filter of rectangular shape is assumed. Remember that $y$ is related to $\vartheta$ via (6). The approximation in (52) is justified by the fact that the phase of the integrand is determined by the high frequency Doppler signal $g\left(y-y^{\prime}\right)$. The antenna pattern $\mathbf{a}(\vartheta)$ can therefore be regarded as constant and drawn in front of the integral.

The choice of the weight vector $\mathbf{w}$ is restricted to the beamforming approaches already presented in Section IV-B.

The DBF method according to (24) can be adapted to the azimuth case by putting a " 1 " to the channel of interest and setting every other channel to zero, giving for example

$$
\mathbf{w}\left(k_{y}\right)=\left[\begin{array}{ll}
1 & 0 \ldots 0
\end{array}\right]^{\mathrm{T}} .
$$

This is repeated for every azimuth wavenumber $k_{y}$. Consequently the azimuth signal spectrum is modulated according to the envelope of the azimuth patterns, as presented in Fig. 24. Of course this spectrum is superimposed by the ambiguous signal spectra. Considering this approach it makes no sense to use more than one channel since the other channels would contaminate the signal of interest with ambiguous signal power for low azimuth sampling wavenumbers $K_{y}$. 
The second method is the MVDR beamformer according to (29)

$$
\mathbf{w}^{*}\left(k_{y}\right)=\frac{\mathbf{R}_{\mathbf{u}}^{-1}\left(k_{y}\right) \mathbf{a}(\vartheta)}{\mathbf{a}^{\mathrm{H}}(\vartheta) \mathbf{R}_{\mathbf{u}}^{-1}\left(k_{y}\right) \mathbf{a}(\vartheta)} .
$$

The angle $\vartheta$ is related to the azimuth wavenumber $k_{y}$ via (42). This beamformer minimizes the additive noise contributions over the wavenumber spectrum generating therefore the optimal SNR. However, since this beamformer only considers the direction of interest, the ambiguous spectra are not suppressed. In the worst case they might even be amplified.

As indicated by the vertical dotted line in Fig. 13 at a certain wavenumber not only the signal of interest is present but also ambiguous signals. A possible way to suppress these ambiguities is the LCMV beamformer (35) as a function of the wavenumbers

$$
\mathbf{w}^{*}\left(k_{y}\right)=\left(\mathbf{R}_{\mathbf{u}}^{-1} \mathbf{A}\left(\mathbf{A}^{\mathrm{H}} \mathbf{R}_{\mathbf{u}}^{-1} \mathbf{A}\right)^{-1} \mathbf{c}\right)\left(k_{y}\right) .
$$

The ambiguous directions $\vartheta_{j}$ are dictated by the integer multiples of the sampling wavenumber $K_{y}$

$$
k_{y}+j K_{y}=-2 k \sin \vartheta_{j}, \quad j \in \mathbb{Z} \backslash\{0\} .
$$

The number of constraints is limited to the number of feed elements in azimuth.

The performance figure quantifying the aliasing effect is the so-called azimuth ambiguity to signal ratio (AASR). This quotient relates ambiguous signal power to signal power according to

$$
\operatorname{AASR}\left(K_{y}\right)=\frac{\sum_{j \in \mathbb{Z} \backslash\{0\}} \int_{-K_{y}^{\prime} / 2}^{K_{1}^{\prime} / 2}\left(\left|\mathbf{w}^{\mathrm{T}} \mathbf{s}\right|^{2}\right)\left(k_{y}+j K_{y}\right) \mathrm{d} k_{y}}{\int_{-K_{y}^{\prime} / 2}^{K_{y}^{\prime} / 2}\left(\left|\mathbf{w}^{\mathrm{T}} \mathbf{S}\right|^{2}\right)\left(k_{y}\right) \mathrm{d} k_{y}} .
$$

The ambiguity expression is independent from the azimuth position on ground $y^{\prime}$, since the reflectivity function $f\left(y^{\prime}\right)$ cancels out. Often in the literature a version of the AASR is presented where the signal spectrum $\mathbf{s}\left(k_{y}\right)$ is replaced by the antenna pattern $\mathbf{a}\left(k_{y}\right)$ [25]. This can be done if the denominator in (41) is neglected. Therefore, this approximation is a worst case estimate for the AASR.

\section{SYSTEM PERFORMANCE}

The following sections are dedicated to the performance analysis in elevation and in azimuth. The analysis is based on the reflector system presented in Section II-B utilizing the DBF algorithms described in Section IV. The graphs presented in this article are the results of numerical simulations.

\section{A. Elevation Performance}

The main performance figure in elevation is the SNR according to (20) for the three DBF approaches, that are the unity weighting method (24), the MVDR beamformer (29), and the LCMV algorithm (35). In

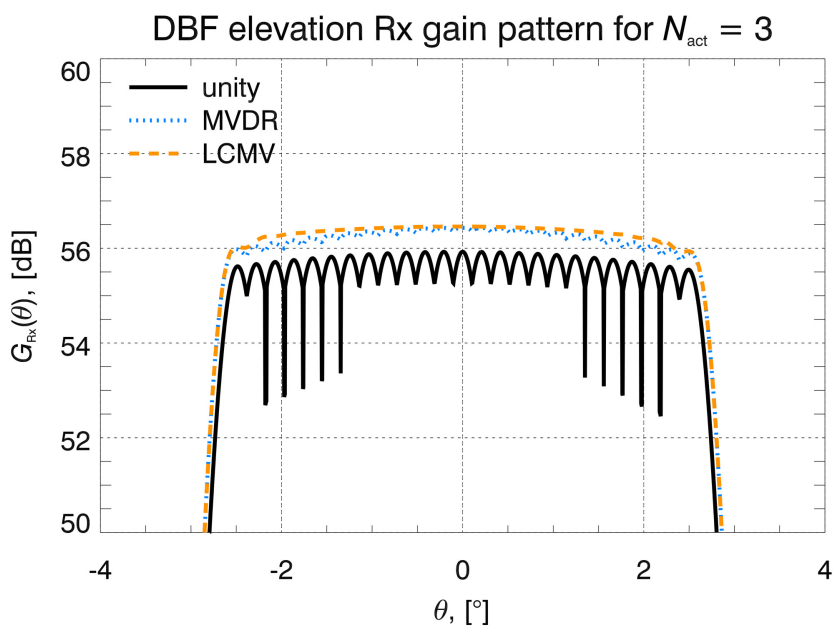

Fig. 14. DBF gain pattern on receive in elevation for three active elements at time instance versus scan angle.

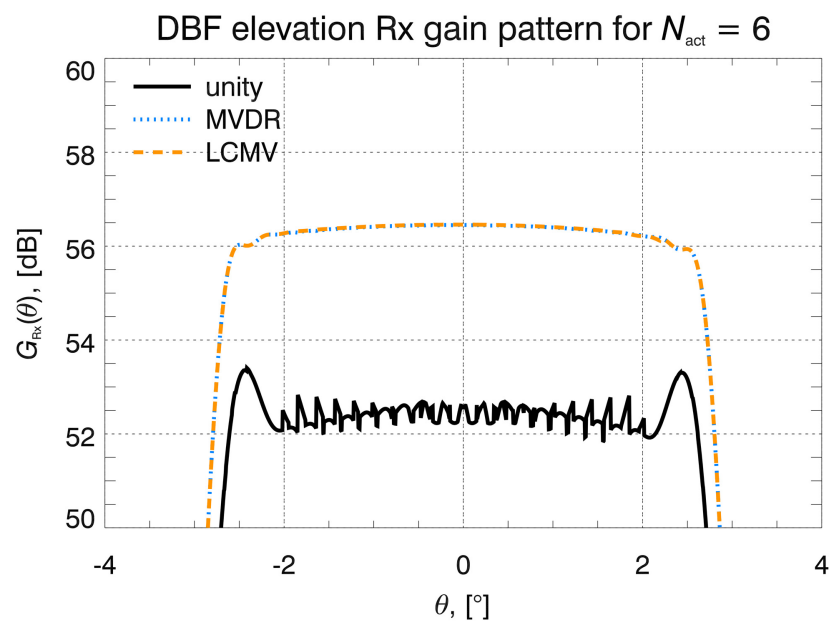

Fig. 15. DBF gain pattern on receive in elevation for six active elements at a time instance.

principle two sources for SNR degradation in the beamforming process can be identified. The first source is spatial mismatch which basically occurs with the unity beamformer (24). What this means can be observed in Fig. 14 and Fig. 15. Here the gain on receive after DBF is presented. Note that $\theta$ in the plot is the same as the scan angle $\vartheta$. In Fig. 14 for the unity approach as well as for the MVDR method always three feed elements are active at a time instance. Only the LCMV beamformer requires all available channels in order to suppress range ambiguities properly. The difference between the LCMV curve and the MVDR curve or the unity beamformer curve can be regarded as SNR loss due to spatial mismatch. Clearly when activating more channels at a time, as shown in Fig. 15, the unity beamformer SNR drops proportional to the number of activated feed elements as predicted by (26). This is because only two or three channels contribute with signal power at a time instance. The additional channels contain noise. In contrast the 


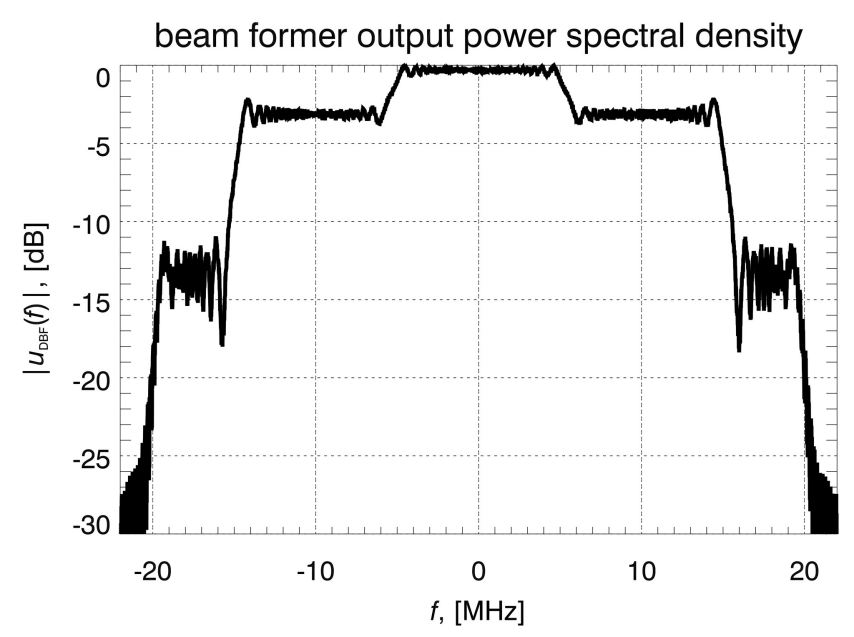

Fig. 16. Unity beamformer output spectrum for point target at swath center.

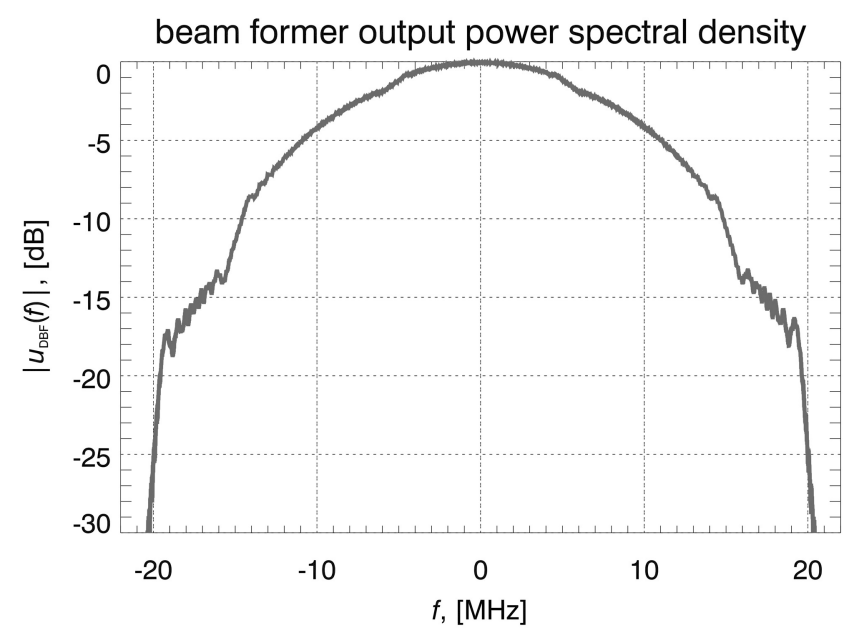

Fig. 17. MVDR beamformer output spectrum for point target at swath center.

MVDR beamformer gains in SNR since more signal power is combined coherently, while noise-only channels are damped with small magnitude weights. In Fig. 15 the LCMV curve overlays the MVDR curve because using more than six elements at a time does not improve the gain significantly more. The discontinuities in the curves for the unity beamformer are a consequence of the channel switching in order to follow the pulse on ground. This analysis shows that for infinitesimal short pulses the SNR loss for the unity approach is on the order of $0.7 \mathrm{~dB}$ assuming $N_{\text {act }}=3$.

The second source of SNR degradation is temporal mismatch. Any real waveform is extended in time in order to emit the available energy. Neglecting this fact by using Dirac-like filters (23) will have a significant impact especially on long duration pulses. Figure 16 and Fig. 17 show the simulated beamformer output spectra of a point target in the swath center $\left(\vartheta=0^{\circ}\right)$. Due to the linear time-frequency dependency of chirp signals the channel switching can be clearly observed

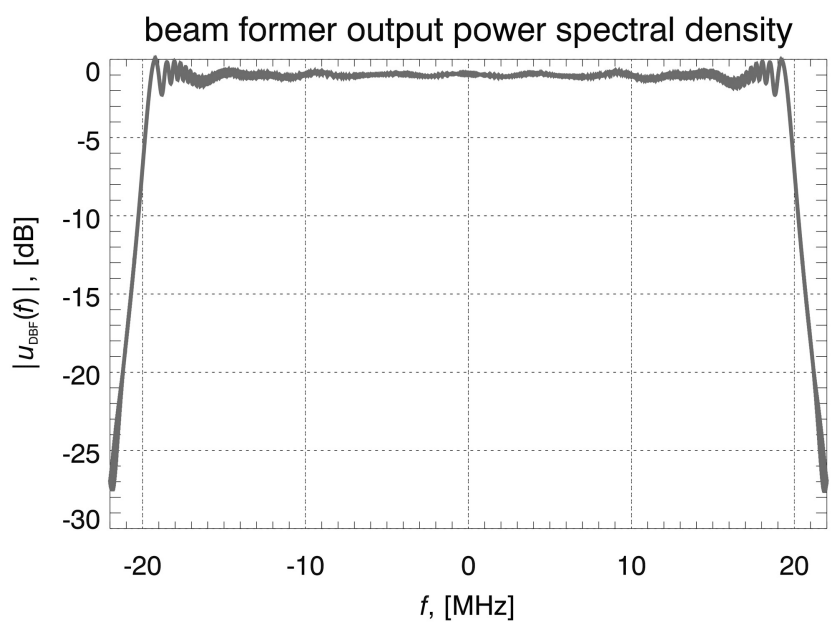

Fig. 18. MVDR beamformer output spectrum using a FIR filter with nine subbands and 31 coefficients.

TABLE II

SNR Loss for the DBF Approaches

\begin{tabular}{ccc}
\hline \hline DBF Method & Dirac-Like Filter & FIR Filter \\
\hline unity & $4.014 \mathrm{~dB}$ & $0.679 \mathrm{~dB}$ \\
MVDR & $4.497 \mathrm{~dB}$ & $0.005 \mathrm{~dB}$ \\
LCMV & $4.554 \mathrm{~dB}$ & $0.001 \mathrm{~dB}$ \\
\hline
\end{tabular}

in the spectra. The spatial weights $\mathbf{w}$ are matched to the pulse center. The loss of signal power is even more severe with the MVDR approach as well as the LCMV beamformer since the low and high frequency parts of the spectra are weighted with small weights.

Generally the SNR loss is affected by both error sources, spatial and temporal mismatch. Table II lists the total SNR losses for the three DBF approaches for the Dirac-like filtering in the middle column and for a FIR filter architecture with $M=9$ subbands and $N_{\text {coef }}=31$ coefficients in the right column. The pulse length $\tau_{\mathrm{p}}$ is $50 \mu \mathrm{s}$. The loss is relative to the maximum SNR, which is produced by the MVDR beamformer using all channels. The numbers suggest that the SNR loss is mainly driven by temporal mismatch. Using an appropriate FIR filter structure allows to reduce the SNR loss as can be seen in Fig. 18. A slight ripple with nine bumps on the signal spectrum caused by the partition into subbands can be observed. This might be mitigated to a certain degree by increasing the number of subbands. But this will require filters with more coefficients which in turn results in a poorer resolution of the frequencies in time domain. This effect is covered by the uncertainty principle

$$
\Delta t \cdot \Delta f \geq 1 \text {. }
$$

Increasing the bandwidth $\Delta f$ results in a finer time domain resolution $\Delta t$ and vice versa. The uncertainty principle in the space-wavenumber domain is

$$
\Delta y \cdot \Delta k_{y} \geq 2 \pi \text {. }
$$



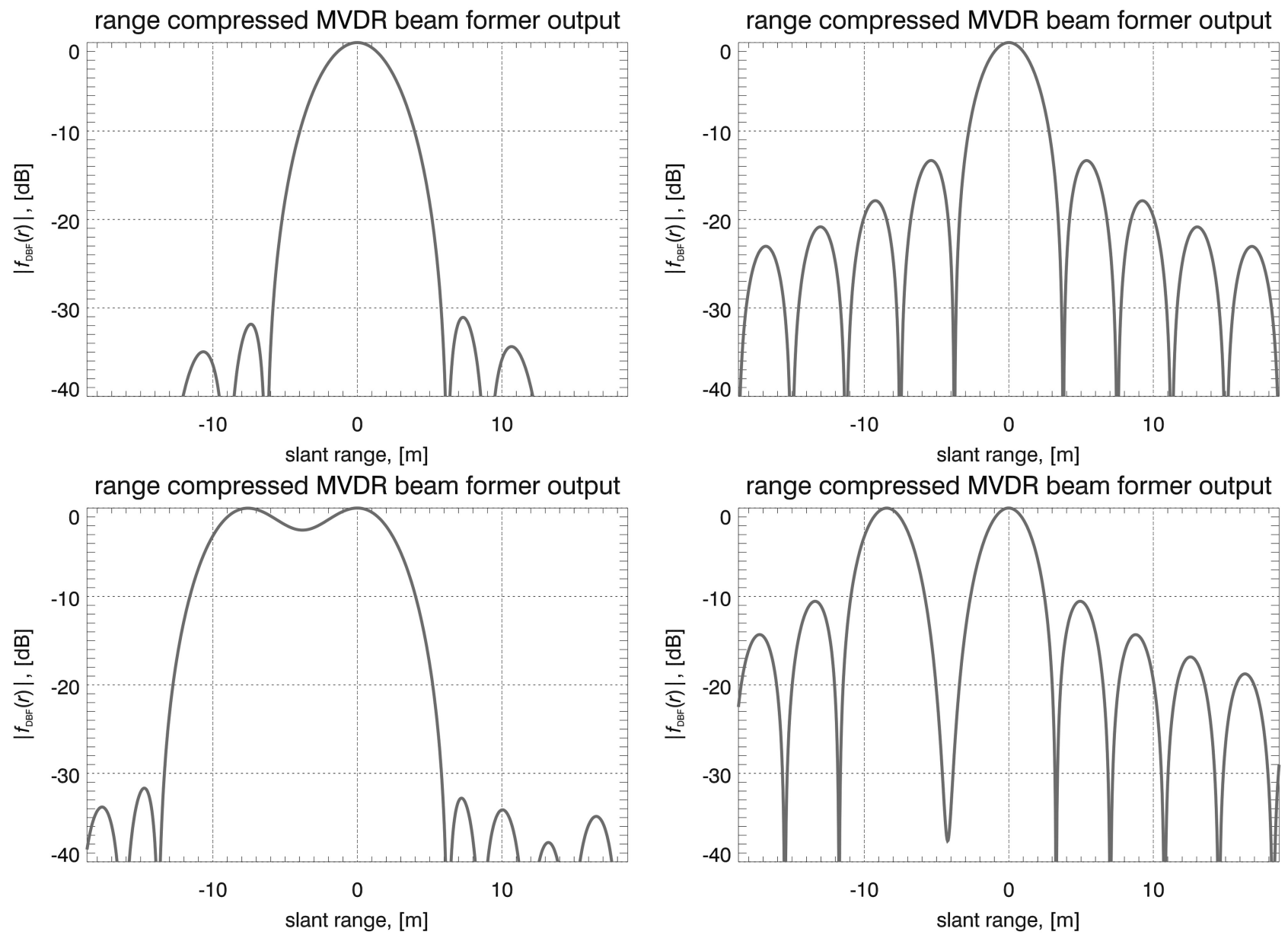

Fig. 19. Upper left: focused point target using MVDR beamformer with Dirac-like filter. Upper right: focused MVDR output using FIR filter with nine subbands and 31 coefficients. Lower row: corresponding simulation to upper row with two adjacent point targets.

The ripple on the signal spectrum could be avoided completely by range compressing the signal prior to spatial weighting. However this approach is computationally most intensive, since filters with thousands of coefficients would be required. But already filters with a relatively low number of coefficients and subbands generate a signal output with consistent gain as demonstrated in Fig. 18.

Figure 19 shows point target simulations using the MVDR beamformer. In the upper left plot a focused point target, with the corresponding spectrum shown in Fig. 17, applying no temporal filtering, is depicted. Clearly the broadening of the mainlobe and the decrease of the sidelobes due to the taper can be observed. In the upper right image a FIR filter with nine subbands and 31 coefficients was used. The corresponding point target spectrum is shown in Fig. 18. In the lower row the simulation results for two point targets in close vicinity are presented. In the lower left image the point targets can hardly be discriminated.

Another important performance measure for a SAR system in elevation is the RASR (36). Since the reflector is quite large in diameter the sidelobes drop quickly moving away from the main beam. This means such a reflector system will inherently have a very good range ambiguity suppression. Therefore it is possible to relax the requirements in the antenna design. For example the elevation height of the antenna could be reduced by employing an elliptical reflector. In the following figures the RASR as a function of the PRF and over the elevation scan angle for the three DBF approaches is presented. Here timing issues are neglected, since emphasis is laid on the beamformer performance. In a real application the timing constraints will display certain PRFs and certain scan angles inaccessible. Figure 20 shows the RASR for the unity beamformer revealing the typical discontinuities in elevation due to the switching. The dynamic range is roughly between $-8 \mathrm{~dB}$ and $-88 \mathrm{~dB}$ for most PRFs. The MVDR method, depicted in Fig. 21, amplifies range ambiguities for certain scan angles and PRFs. Comparing the MVDR RASR to the unity beamforming result, the shape of the plot seems to be preserved. However the RASR for the LCMV method is improved substantially as can be seen in Fig. 22. This becomes evident in the DBF receive pattern plot shown in Fig. 23. Here a high 


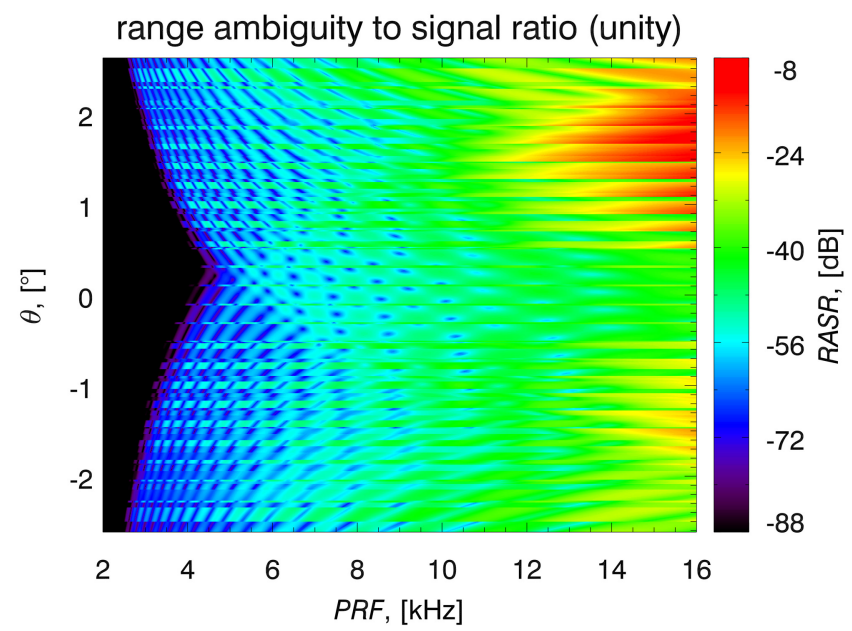

Fig. 20. RASR for unity beamformer.

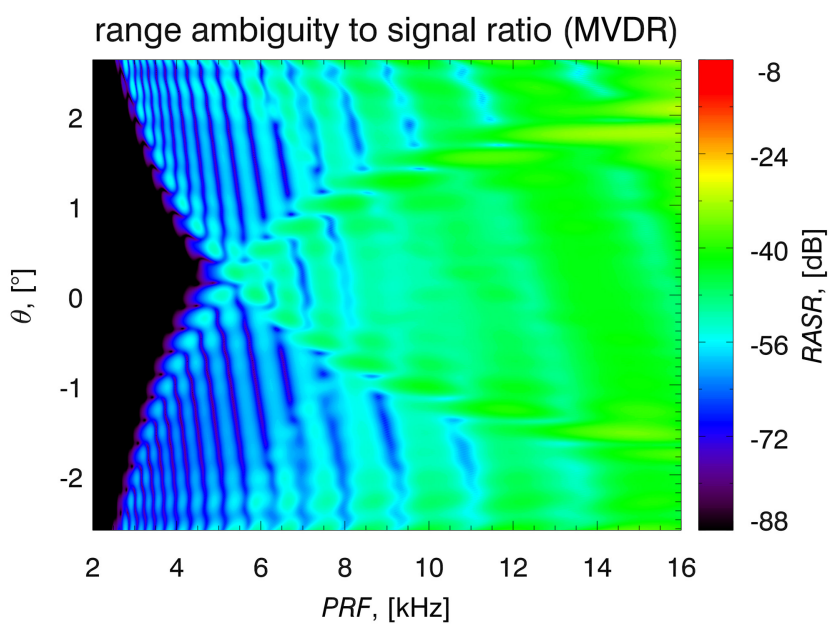

Fig. 21. RASR for MVDR beamformer.

PRF of $13.5 \mathrm{kHz}$ is chosen in order to demonstrate the capabilities of the LCMV beamformer. There is of course an absolute limit where the LCMV beamformer will work, that is the minimum beamwidth to be generated with this antenna. Ambiguous directions cannot be suppressed if they enter the main beam. The scan angle for the signal of interest, marked by the vertical dashed line, is $1.36^{\circ}$. At this angle the RASR for the unity beamformer as well as the MVDR approach is relatively poor. The ambiguous directions, indicated by the vertical solid lines in Fig. 23 are damped quite well with the LCMV method while the gain loss is negligible.

In some cases the range ambiguities are not suppressed well. This is a result of an improper selection of the aforementioned eigenvalue threshold parameter.

\section{B. Azimuth Performance}

The performance in azimuth is characterized by the SNR given with (50) and the AASR defined in (57). The SNR is evaluated in the same manner as in the elevation case. A point target was simulated

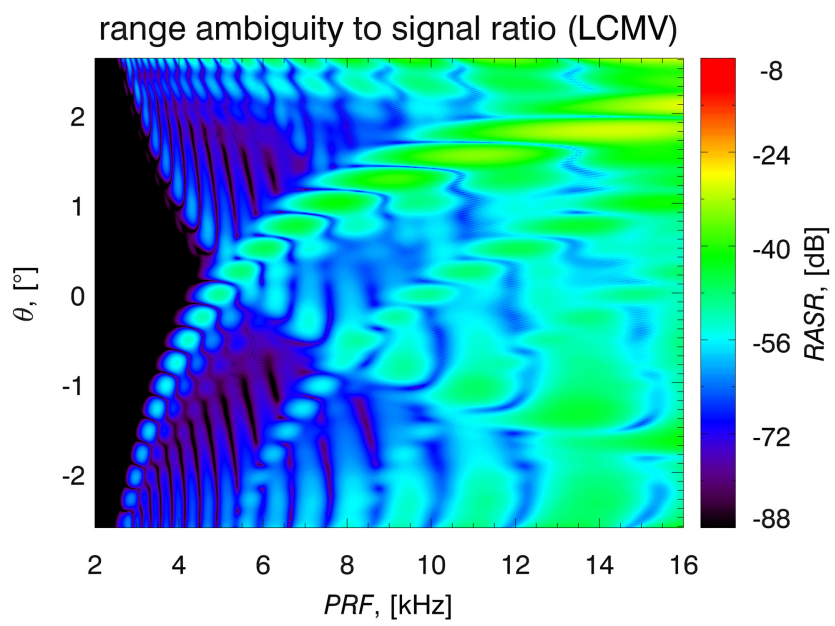

Fig. 22. RASR for LCMV beamformer.

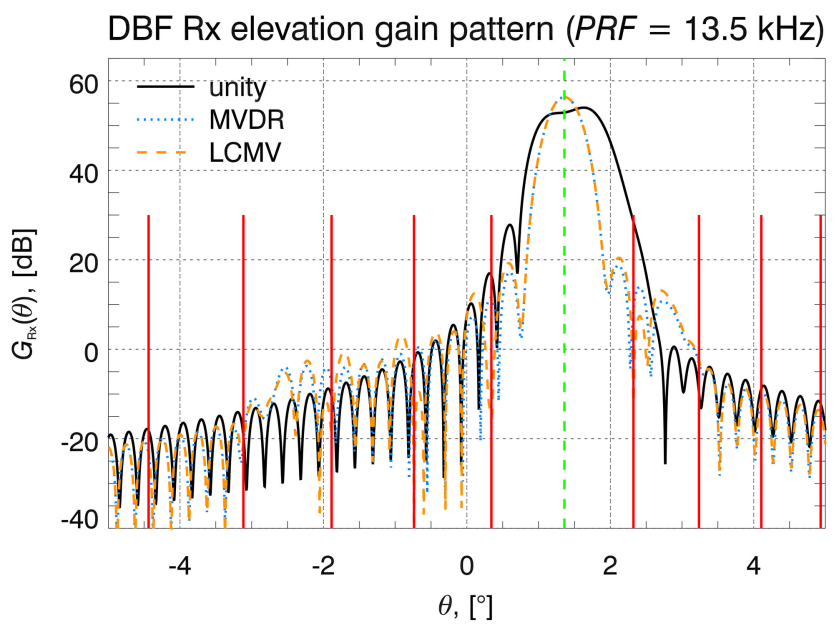

Fig. 23. DBF receive pattern in elevation for the three beamformers with position of signal (vertical dashed line) and position of the range ambiguities (vertical solid lines).

TABLE III

SNR Loss for DBF Approaches

\begin{tabular}{cc}
\hline \hline DBF Method & SNR Loss \\
\hline unity & $3.06 \mathrm{~dB}$ \\
MVDR & $0.00 \mathrm{~dB}$ \\
LCMV & $1.39 \mathrm{~dB}$ \\
\hline
\end{tabular}

in the swath center and processed using the three DBF approaches. Beamforming in azimuth is simpler insofar as no temporal filtering is required. Table III summarizes the SNR losses with respect to the optimal beamformer, which is the MVDR beamformer. Since the unity beamformer uses only one channel per Doppler wavenumber, which is basically the channel with the highest gain, the loss is $3 \mathrm{~dB}$. This can be clarified via the channel patterns in Fig. 24 at wavenumber zero. Only one of the two center beams is used which means a signal loss of $3 \mathrm{~dB}$. From this plot the maximum achievable azimuth resolution can be found with (59) to be on 


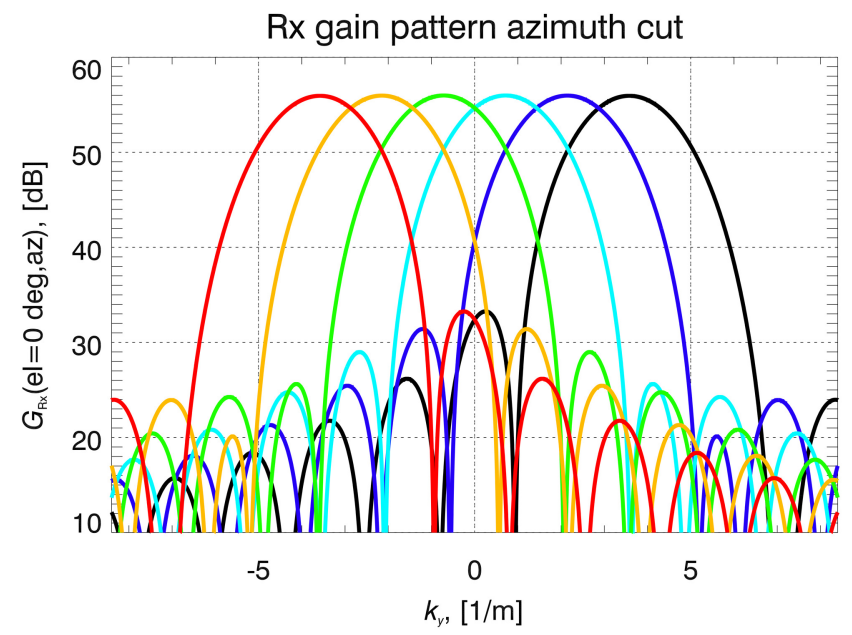

Fig. 24. Azimuth channel pattern versus wavenumber.

the order of $0.67 \mathrm{~m}$ using the full $3 \mathrm{~dB}$ bandwidth of $9.4 \mathrm{~m}^{-1}$. However for this simulation a processed azimuth bandwidth $B_{y}$ of $10 \mathrm{kHz}$, corresponding to $K_{y}^{\prime}=8.4 \mathrm{~m}^{-1}$, was used, yielding a resolution of approximately $0.75 \mathrm{~m}$. Figure 25 shows the results of point target simulations for the three beamformers. All DBF approaches result in the typical sinus cardinalis shape of the point target response with the predicted resolutions.

The azimuth bandwidth impacts directly the AASR. Figure 26 shows the AASR as a function of the PRF. The curve for the LCMV beamformer starts at a PRF of ca. $2.6 \mathrm{kHz}$, since for lower PRFs the azimuth ambiguities enter the main beam. Obviously the MVDR beamformer amplifies azimuth ambiguities for a wide range of PRFs. This effect can be observed in the corresponding pattern plot in Fig. 27. The signal, marked by the vertical dashed line, is at Doppler frequency zero. The ambiguous Doppler frequencies, indicated by the vertical solid lines, occur at multiples of the PRF away from the signal frequency. The unity beamformer uses a single channel receiving the main amount of ambiguous signal power from the Doppler frequency $3 \mathrm{kHz}$. The MVDR beamformer produces the highest gain at the cost of ambiguous signal power from Doppler frequency $\pm 3 \mathrm{kHz}$. Only the LCMV beamformer is able to suppress both ambiguities efficiently.
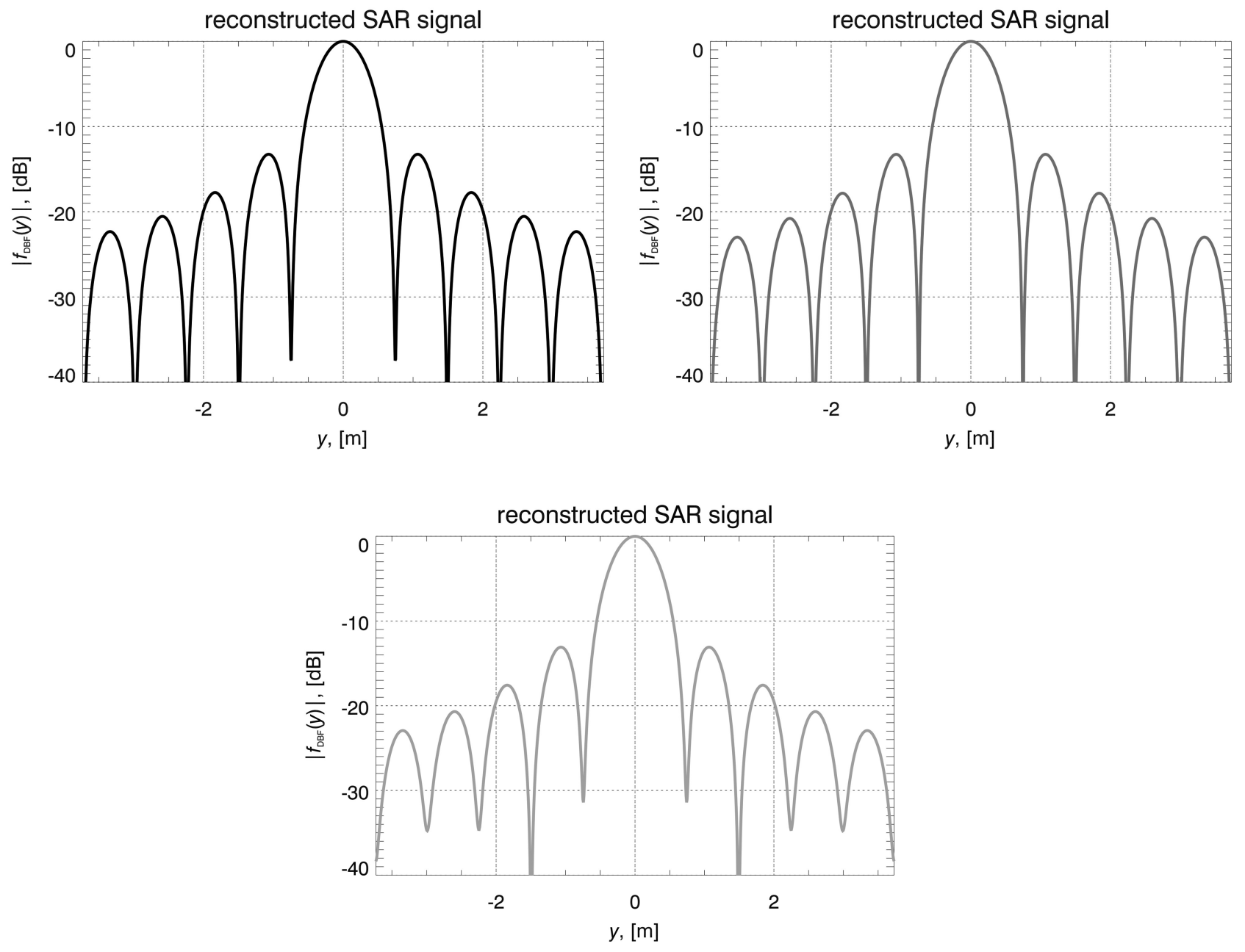

Fig. 25. Upper left: focused point target using unity DBF. Upper right: focused point target using MVDR DBF. Lower image: focused point target using LCMV DBF. 


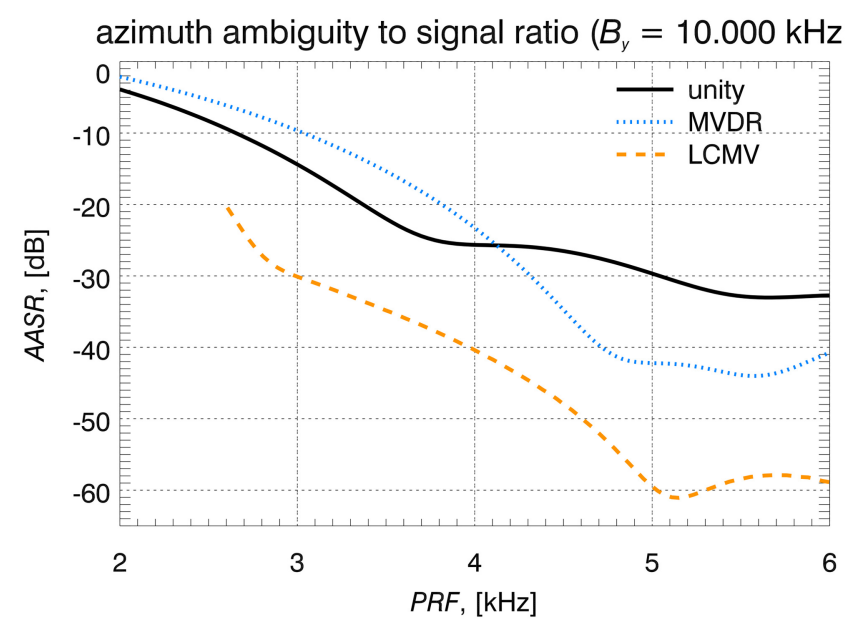

Fig. 26. AASR for the three DBF approaches versus PRF taken at swath center.

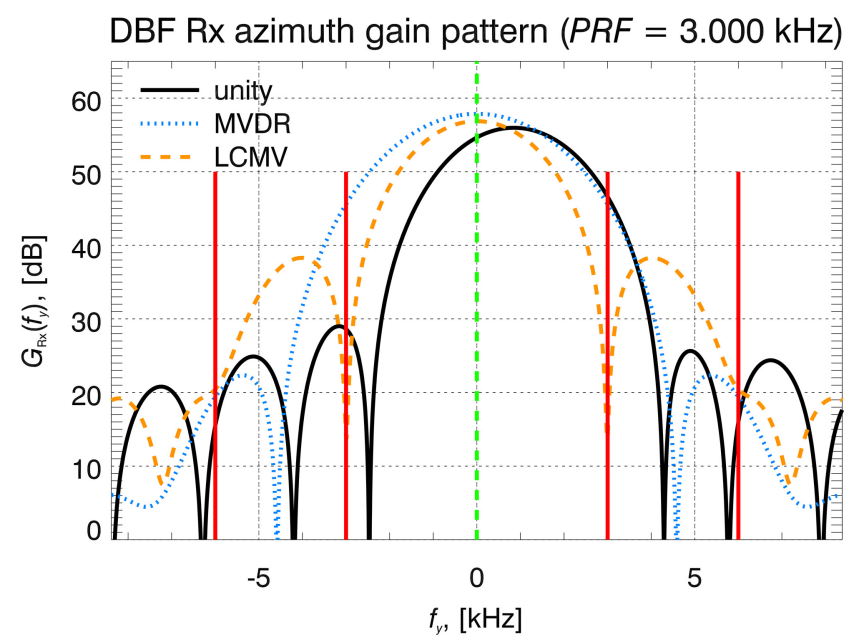

Fig. 27. Rx gain pattern in azimuth Doppler frequency domain with position of signal (vertical dashed line) and position of azimuth ambiguities (vertical solid lines).

\section{CONCLUSION}

In the frame of SAR imaging a detailed analysis of the innovative concept of combining a parabolic reflector with a digital feed array was presented. The inherent focusing capabilities of reflector antennas provide an excellent performance especially in elevation. However in order to operate such systems with long duration pulses, more sophisticated beamforming approaches are of vital importance. It was shown that with FIR filter structures in conjunction with spatially matched coefficients, that is the MVDR beamformer, the SNR loss can be drastically reduced. In the presence of range ambiguities the LCMV beamformer offers the opportunity to improve the SAR image quality further. The LCMV beamformer shows its full potential when going for high azimuth resolution imagery. The AASR, being the main source of degradation, was improved significantly.
The investigations in this article are based on perfect knowledge of all parameters involved in the beamforming concepts. The most important parameter is definitely the complex antenna pattern. Since the DOA is only known to a certain degree and the pattern measurements are subject to various error sources, the performance can be expected to degrade. Therefore in future investigations the impact of these error sources will be investigated with the goal of improving the robustness of potential beamforming methods.

\section{APPENDIX. SIGNAL-TO-NOISE RATIO}

The SNR is derived in time domain with the discrete time variable $n$. Rewriting the beamformer output for a point target gives

$$
\begin{aligned}
u_{\mathrm{DBF}}(n)= & \sum_{i} \sum_{n^{\prime}} u_{i}\left(n-n^{\prime}\right) h_{i}\left(n, n^{\prime}\right) \\
= & \sum_{i} \sum_{n^{\prime}} f(\vartheta) a_{i}(\vartheta) g\left(\vartheta, n-n^{\prime}\right) h_{i}\left(n, n^{\prime}\right) \\
& +\sum_{i} \sum_{n^{\prime}} v_{i}\left(n-n^{\prime}\right) h_{i}\left(n, n^{\prime}\right) .
\end{aligned}
$$

Note that $f$ as well as $v_{i}$ are complex random processes. If we assume uniform and independent Gaussian random variables for the real part and the imaginary part with zero mean, respectively, the processes are characterized by their variances $\sigma_{f}^{2}$ and $\sigma_{v_{i}}^{2}$. Assuming uncorrelated noise and signal, the power of the beamformer output is

$$
\begin{aligned}
P_{u_{\mathrm{DBF}}}(n)= & \mathrm{E}\left\{\left|u_{\mathrm{DBF}}(n)\right|^{2}\right\} \\
= & \mathrm{E}\left\{\left|\sum_{i} \sum_{n^{\prime}} f(\vartheta) a_{i}(\vartheta) g\left(\vartheta, n-n^{\prime}\right) \times h_{i}\left(n, n^{\prime}\right)\right|^{2}\right\} \\
& +\mathrm{E}\left\{\left|\sum_{i} \sum_{n^{\prime}} v_{i}\left(n-n^{\prime}\right) h_{i}\left(n, n^{\prime}\right)\right|^{2}\right\} \\
= & P_{s}(n)+P_{v}(n)
\end{aligned}
$$

with $\mathrm{E}\{$.$\} the expectation operator. In principle$ the SNR can already be computed. However this expression is difficult to interpret in the traditional sense of DBF. Exchanging the summation between $n^{\prime}$ and $i$ the signal power $P_{s}(n)$ can be written in vector notation as

$$
\begin{aligned}
P_{s}(n) & =\mathrm{E}\left\{\left|\sum_{n^{\prime}} f(\vartheta) g\left(\vartheta, n-n^{\prime}\right) \times \mathbf{a}^{\mathrm{T}}(\vartheta) \mathbf{h}\left(n, n^{\prime}\right)\right|^{2}\right\} \\
& =\sigma_{f}^{2}(\vartheta)\left|\mathbf{a}^{\mathrm{T}}(\vartheta) \sum_{n^{\prime}} g\left(\vartheta, n-n^{\prime}\right) \mathbf{h}\left(n, n^{\prime}\right)\right|^{2} .
\end{aligned}
$$


Similarly the noise power $P_{v}(n)$ can be expressed as

$$
P_{v}(n)=\mathrm{E}\left\{\left|\sum_{n^{\prime}} \mathbf{h}^{\mathrm{T}}\left(n, n^{\prime}\right) \mathbf{v}\left(n-n^{\prime}\right)\right|^{2}\right\} .
$$

In order to account for all combinations when evaluating the squared expression $|.|^{2}$ in (67) another variable $m^{\prime} \in\left[0, N_{\text {coef }}-1\right]$ is introduced. This gives

$$
\begin{aligned}
P_{v}(n)= & \sum_{n^{\prime}} \sum_{m^{\prime}} \mathbf{h}^{\mathrm{T}}\left(n, n^{\prime}\right) \mathrm{E}\left\{\mathbf{v}\left(n-n^{\prime}\right)\right. \\
& \left.\times \mathbf{v}^{\mathrm{H}}\left(n-m^{\prime}\right)\right\} \mathbf{h}^{*}\left(n, m^{\prime}\right) \\
= & \sum_{n^{\prime}} \sum_{m^{\prime}} \mathbf{h}^{\mathrm{T}}\left(n, n^{\prime}\right) \\
& \times \mathbf{R}_{\mathbf{v}}\left(n-n^{\prime}, n-m^{\prime}\right) \mathbf{h}^{*}\left(n, m^{\prime}\right) .
\end{aligned}
$$

A simplification can be introduced if one assumes that the samples of the random processes are mutually independent. Then the covariance matrices become the zero matrices for $n^{\prime} \neq m^{\prime}$ and the noise power is

$$
P_{v}(n)=\sum_{n^{\prime}} \mathbf{h}^{\mathrm{T}}\left(n, n^{\prime}\right) \mathbf{R}_{\mathbf{v}}\left(n-n^{\prime}\right) \mathbf{h}^{*}\left(n, n^{\prime}\right) .
$$

For a Dirac impulse response function $h_{i}\left(n, n^{\prime}\right)=$ $w_{i}(n) \delta\left(n^{\prime}\right)$ this is the well-known expression of the noise power for a multi-channel beamformer. Finally this expression can be further simplified, if channels with cross powers zero are assumed, yielding

$$
P_{v}(n)=\sum_{i} \sigma_{v_{i}}^{2} \sum_{n^{\prime}}\left|h_{i}\left(n, n^{\prime}\right)\right|^{2} .
$$

The SNR for the compressed SAR signal can then be computed as

$$
\operatorname{SNR}(n) \approx \frac{\left|\sum_{n}^{n+N_{\mathrm{p}}} \sqrt{P_{s}(n)}\right|^{2}}{\sum_{n}^{n+N_{\mathrm{p}}} P_{v}(n)}
$$

substituting (66) for the signal power and either (69), (70), or (71) for the noise power.

It is important to mention that the SNR expression (72) is only valid if the sampling frequency $f_{\mathrm{s}}$ is smaller than the signal bandwidth $B$. If the sampling frequency is larger than the bandwidth the noise samples become dependent and combine therefore coherently as is the case for the signal samples. Principally the SNR cannot be improved by oversampling.

\section{REFERENCES}

[1] Krieger, G., et al. Tandem-L: An innovative interferometric and polarimetric SAR mission to monitor Earth system dynamics with high resolution.

In Proceedings of the IEEE Geoscience and Remote Sensing Symposium (IGARSS), July 2010, pp. 253-256.

[2] Currie, A. and Brown, M.

Wide-swath SAR.

IEE Proceedings $-F$ Radar and Signal Processing, 139, 2 (Apr. 1992), 122-135.
[3] Li, Z., et al.

Performance improvement for constellation SAR using signal processing techniques.

IEEE Transactions on Aerospace and Electronic Systems, 42, 2 (Apr. 2006), 436-452.

[4] Krieger, G., et al.

Advanced concepts for ultra-wide-swath SAR imaging. In Proceedings of the European Conference on Synthetic Aperture Radar (EUSAR), vol. 2, June 2008, pp. 31-34.

[5] Krieger, G., et al.

Advanced synthetic aperture radar based on digital beamforming and waveform diversity.

In Proceedings of the IEEE Radar Conference (RADAR), May 2008, pp. 767-772.

[6] Younis, M., et al.

Performance comparison of reflector- and planar-antenna based digital beam-forming SAR.

International Journal of Antennas and Propagation, 2009 (June 2009), 1-14.

[7] Freeman, A., et al.

SweepSAR: Beamforming on receive using a

reflector-phased array feed combination for spaceborne SAR.

In Proceedings of the IEEE Radar Conference (RadarCon), May 2009.

[8] Huber, S., et al.

A novel digital beam-forming concept for spaceborne reflector SAR systems.

In Proceedings of the European Radar Conference (EuRAD), Oct. 2009, pp. 238-241.

[9] Huber, S., et al.

Digital beam forming concepts with application to spaceborne reflector SAR systems.

In Proceedings of the International Radar Symposium (IRS), June 2010, pp. 1-4.

[10] Huber, S., et al.

Digital beam forming techniques for spaceborne reflector SAR systems.

In Proceedings of the 8th European Conference on Synthetic Aperture Radar (EUSAR), June 2010, pp. 962-965.

[11] General reflector and antenna farm analysis software (Aug. 2011).

[Online], available: http://www.ticra.com/.

[12] Milligan, T. A.

Modern Antenna Design (2nd ed.).

Hoboken, NJ: Wiley, 2005.

[13] von Hoerner, $\mathrm{S}$

Strong coma lobes from small gravitational deformations. IEEE Transactions on Antennas and Propagation, AP-28, 5 (Sept. 1980), 652-657.

[14] Blythe, J. H.

Radar systems.

U.S. Patent 4253 098, Feb. 1981.

[15] Kare, J. T.

Moving receive beam method and apparatus for synthetic aperture radar.

U.S. Patent 6175 326, Jan. 2001.

[16] Suess, M., Grafmueller, B., and Zahn, R. A novel high resolution, wide swath SAR.

In Proceedings of the IEEE 2001 International Geoscience and Remote Sensing Symposium (IGARSS), vol. 3, 2001, pp. 1013-1015.

[17] Suess, M. and Wiesbeck, W.

Side-looking synthetic aperture radar system. European Patent EP 1241 487, Sept. 2002. 
Adaptive scan-on-receive based on spatial spectral estimation for high-resolution, wide-swath synthetic aperture radar.

In Proceedings of the IEEE International Geoscience and Remote Sensing Symposium (IGARSS), vol. 1, July 2009, pp. 64-67.

Patyuchenko, A., et al.

Performance optimization of the reflector antenna for the digital beam-forming SAR system.

In Proceedings of Advanced RF Sensors and Remote Sensing Instruments (ARSI), 2009.

Capon, J.

High-resolution frequency-wavenumber spectrum analysis.

Proceedings of the IEEE, 57, 8 (Aug. 1969), 1408-1418.
Robust minimum variance beamforming. IEEE Transactions on Signal Processing, 53, 5 (May 2005), 1684-1696.

[23] Freeman, A., et al.

The 'myth' of the minimum SAR antenna area constraint. IEEE Transactions on Geoscience and Remote Sensing, 38, 1 (Jan. 2000), 320-324.

[24] Gebert, N., Krieger, G., and Moreira, A.

Digital beamforming on receive: Techniques and optimization strategies for high-resolution wide-swath SAR imaging.

IEEE Transactions on Aerospace and Electronic Systems, 45, 2 (Apr. 2009), 564-592.

[25] Curlander, J. C. and McDonough, R. N. Synthetic Aperture Radar Systems and Signal Processing. Hoboken, NJ: Wiley, 1991.
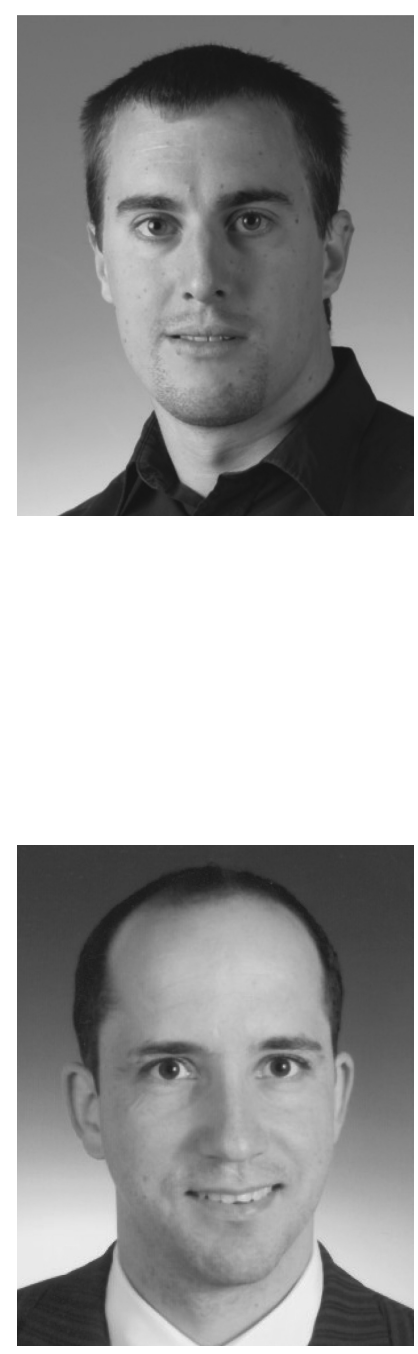

Sigurd Huber received the Dipl.-Ing. (M.S.) degree in electrical and communication engineering from the Technical University of Munich (TUM), Germany, in 2005.

Since 2005 he has worked as a scientist at the Microwaves and Radar Institute of the German Aerospace Center (DLR), Oberpfaffenhofen, Germany. He is involved in national and international projects in the field of synthetic aperture radar, encompassing cooperations with industry partners (EADS Astrium), as well as research organizations (ESA, NASA/JPL). His research interests include digital signal processing, electromagnetic field theory and inverse problems.

Mr. Huber has contributed as author and coauthor in more than 30 conference papers and four peer reviewed journal articles.

Marwan Younis (S'1995-M'2005-SM'2008) was born in Las Cruces, NM, in 1970. He received his B.Sc. in electrical engineering from the University of Baghdad, Iraq in 1992 and the Dipl.-Ing. (M.Sc.) and Dr.-Ing. (Ph.D.) degree in electrical engineering from the Universität Karlsruhe (TH), Germany, in 1997 and 2004, respectively.

From 1998 to 2004, he was a research scientist with the Institut für Höchstfrequenztechnik und Elektronik, Universität Karlsruhe (TH). Since 2005 he has been with the Microwaves and Radar Institute of the German Aerospace Center (DLR), Oberpfaffenhofen, Germany. He is a lecturer with the Universität Karlsruhe (TH) and the Carl-Cranz-Gesellschaft. His research fields include synthetic aperture radar (SAR) systems, SAR performance, digital beamforming for radar, synchronization of bistatic SAR, forward looking radar, and antennas.

Dr. Younis is the author and coauthor of over 70 conference papers and more than 15 reviewed publications. He is an active member of the German Association for Location and Navigation (DGON). He is the active microwave working group leader within the IEEE Instruments and Future Technologies Technical Committee. He received the Hermann-Billing award for his Ph.D. thesis in 2005. 

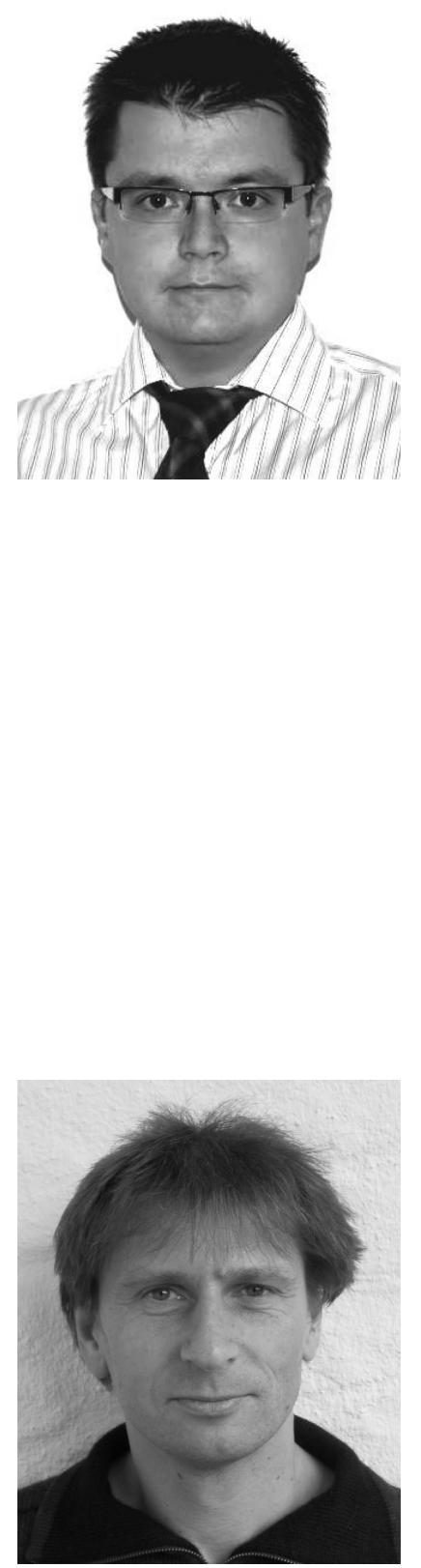

Anton Patyuchenko received his Master degree from the Technical University of Munich, Germany.

$\mathrm{He}$ is a scientific assistant at the Microwaves and Radar Institute (HR) of German Aerospace Center (DLR) and is currently working towards his Ph.D. He has been contributing to various research programmes and projects conducted in collaboration with ESA, Astrium, and NASA JPL, including digital beamforming (HRWS), TanDEM-X, Tandem-L, and other research activities related to future radar remote sensing missions for Earth observation applications. His research areas cover digital beamforming (DBF) SAR, reflector antennas for future spaceborne SAR missions, radar systems for space debris detection.

Mr. Patyuchenko received DAAD/Siemens Scholarship Award in 2005, and special award for personal and professional contribution to TanDEM-X mission (from DLR e.V., Astrium GmbH, and Infoterra GmbH) in 2010.

Gerhard Krieger received the Dipl.-Ing. (M.S.) and Dr.-Ing. (Ph.D.) degrees (with honors) in electrical and communication engineering from the Technical University of Munich, Germany, in 1992 and 1999, respectively.

From 1992 to 1999, he was with the Ludwig-Maximilians University, Munich, where he conducted multidisciplinary research on neuronal modeling and nonlinear information processing in biological and technical vision systems. In 1999, he joined the Microwaves and Radar Institute (HR) of the German Aerospace Center (DLR), Oberpfaffenhofen, Germany, where he developed signal and image processing algorithms for a novel forward looking radar system employing digital beamforming on receive. From 2001 to 2007 he led the New SAR Missions Group which pioneered the development of advanced bistatic and multistatic radar systems as exemplified by the forthcoming TanDEM-X mission as well as innovative multi-channel SAR techniques and algorithms for high-resolution wide-swath SAR imaging. Since 2008, he has been Head of the new Radar Concepts Department of the Microwaves and Radar Institute, DLR, Oberpfaffenhofen, Germany. His current research interests focus on the development of multi-channel radar techniques and algorithms for innovative MIMO SAR systems, the demonstration of novel interferometric and tomographic Earth observation applications, and the conceptual design of advanced bi- and multistatic radar missions.

Dr. Krieger has received several national and international awards, including the W.R.G. Baker Prize Paper Award from the IEEE board of directors and the IEEE Transactions Prize Paper Award of the Geoscience and Remote Sensing Society. He is author of about 50 peer reviewed journal papers, 4 invited book chapters, more than 200 conference papers, and 5 patents. 


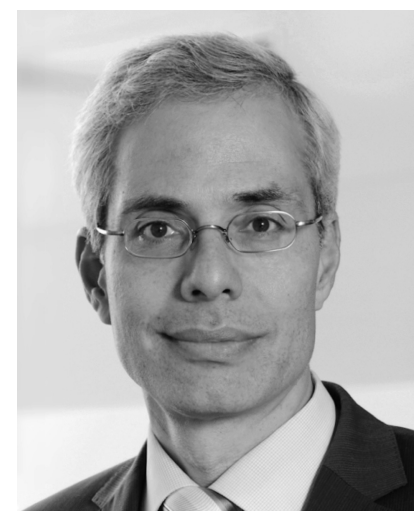

Alberto Moreira (S'96-F'04) received the B.S.E.E. and M.S.E.E. degrees from the Aeronautical Technological Institute (ITA), São José dos Campos, Brazil, in 1984 and 1986, respectively, and the Eng. Dr. degree (with honors) from the Technical University of Munich, Germany, in 1993.

From 1996 to 2001, he was the chief scientist and engineer with the SAR Technology Department, German Aerospace Center (DLR), Oberpfaffenhofen, Germany. Since 2001 he has been the Director of the Microwaves and Radar Institute at DLR. The Institute contributes to several scientific programs and space projects for actual and future airborne and spaceborne SAR missions like TerraSAR-X, TanDEM-X and Sentinel-1. The mission TanDEM-X, led by his Institute, has successfully started the operational phase in December 2010. Professor Moreira is the initiator and principal investigator for this mission. Since 2003, he is also a full professor with the Karlsruhe Institute of Technology, Germany, in the field of microwave remote sensing. His professional interests and research areas encompass radar end-to-end system design and analysis, innovative microwave techniques and system concepts, signal processing, and remote sensing applications.

Dr. Moreira has more than 300 publications in international conferences and journals and is the holder of 15 patents in the radar and antenna field. He is a member of the IEEE Geoscience and Remote Sensing Society (GRSS) Administrative Committee (1999-2001, 2004-2013, 2010 as president, 2011-2013 as past-president), was the Founder and Chair of the GRSS German Chapter (2003-2008) and associate editor for the IEEE Goescience and Remote Sensing Letters (2003-2007) and for the IEEE Transactions on Geoscience and Remote Sensing (2005-2011). He and his colleagues received the GRS-S Transactions Prize Paper Awards in 1997, 2001, and 2007 and the IEEE W.R.G. Baker Award in 2012. He is also the recipient of the DLR Science Award (1995), IEEE Nathanson Award for the Young Radar Engineer of the Year (1999) and the IEEE Kiyo Tomiyasu Field Award (2007). From 2003 until 2008, he served as a member of the Board of Directors of the Information Technology Society of the German Association for Electrical, Electronic and Information Technologies and as Chair of the Scientific and Technical Council of DLR (2009-2011). He has contributed to the successful series of the European SAR conferences (EUSAR) since 1996 as a member of the Technical Program Committee, technical chairman (2000), awards chairman (2002-2004), general chairman (2006), and cochairman (2008) and is serving as general cochair for IGARSS'12 in Munich. 